\title{
Bioethica
}

Vol 6, No 1 (2020)

Bioethica

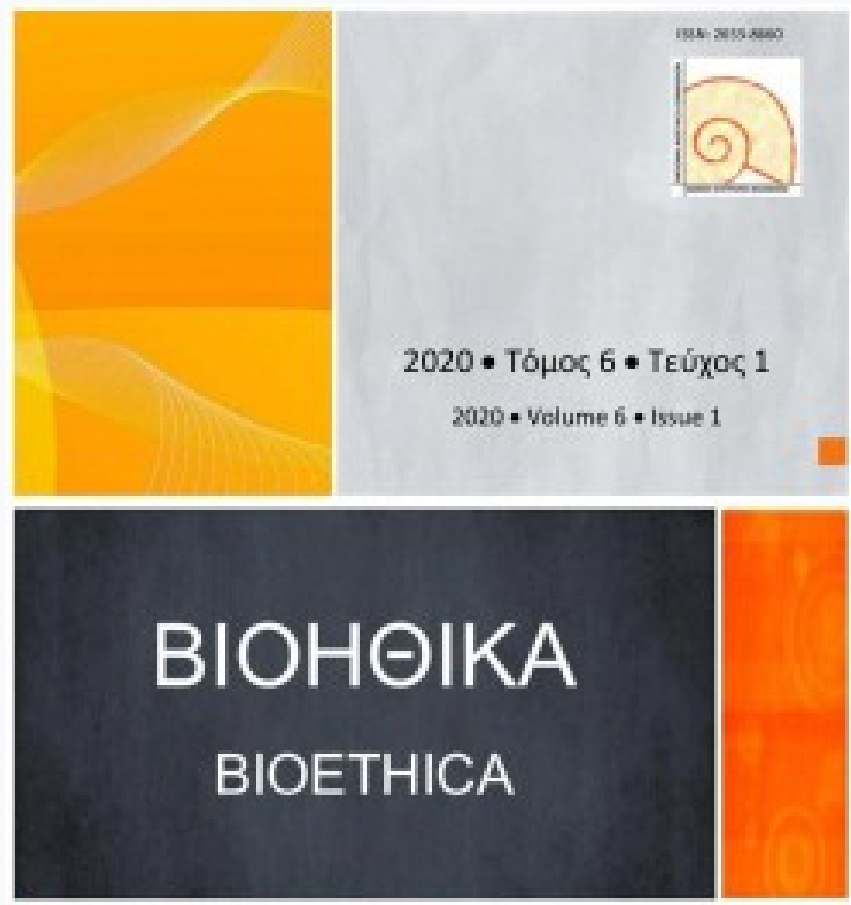

The commercialization of the human body

Maria Merkou (Mapía Мغ́ркоu)

doi: $10.12681 /$ bioeth.22620

Copyright @ 2020, Maria Merkou (Mapía Мع́pкоu)

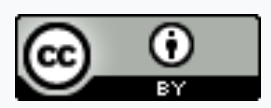

This work is licensed under a Creative Commons Attribution 4.0

To cite this article:

Merkou (Mapía Mćpкоu) M. (2020). The commercialization of the human body. Bioethica, 6(1), 17.

https://doi.org/10.12681/bioeth.22620 


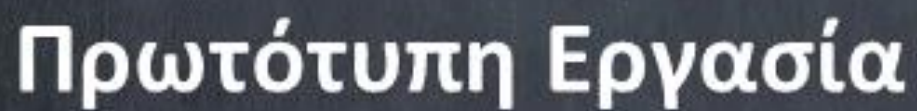

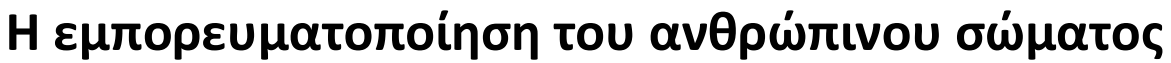

\section{Mapía Мв́ркоu}

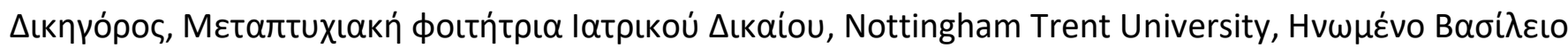

m.merkoy@gmail.com

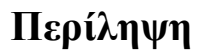

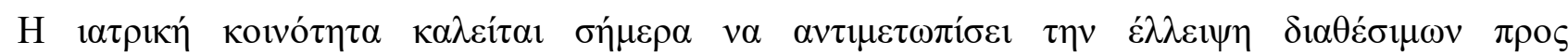

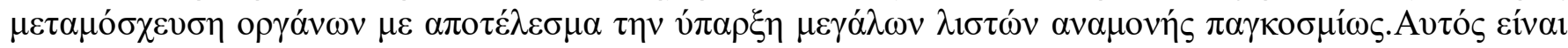

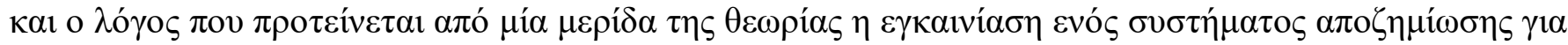

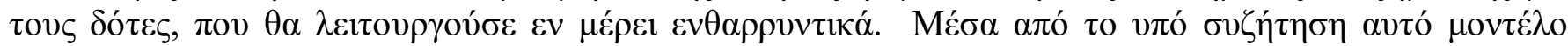

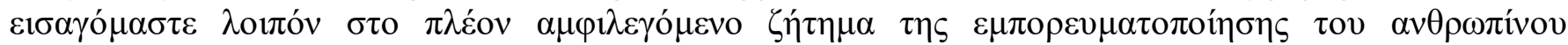

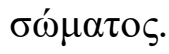

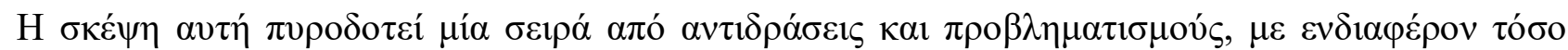

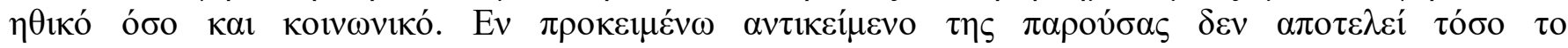

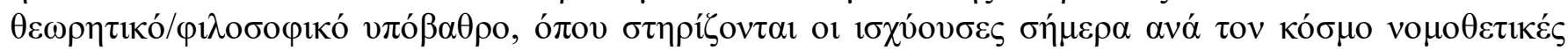

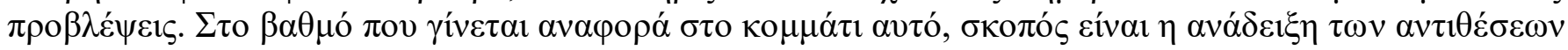

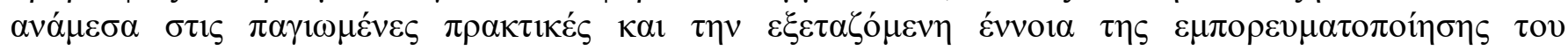

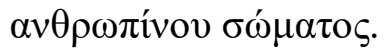

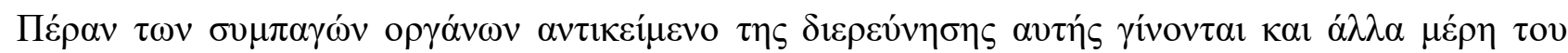

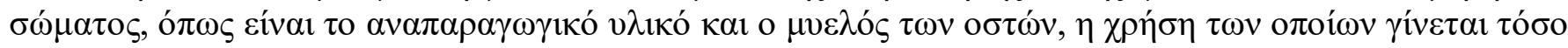

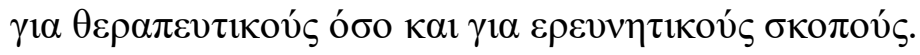

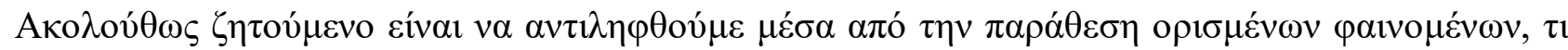

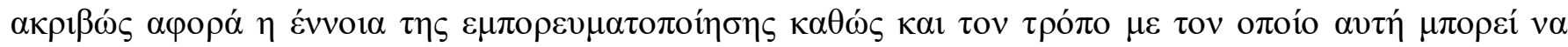

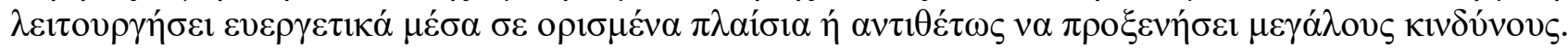

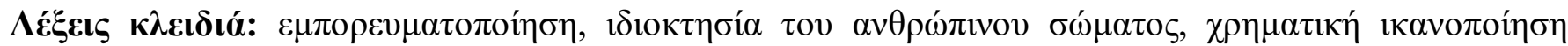

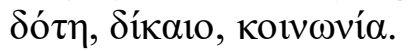




\title{
The commercialization of the human body
}

\section{Maria Merkou}

Lawyer, Postgraduate Student LLM Health Law and Ethics, Nottingham Trent University, United Kingdom

\begin{abstract}
The medical community has to confront the shortage of organs available for human transplantation, resulting in large waiting lists worldwide. Hence, the introduction of a donor reimbursement system, which would partly serve as an incentive is often proposed. This model leads us, however, to the muchdisputed notion of the commercialization of the human body.

This concept triggers a series of reactions of both moral and social interest. However, the philosophical justification of the actual legislative provisions is only used as a means to highlight the conflict between the various practices on the matter and is not itself the main focus of the present essay.

In addition to solid organs, other parts of the body such as the human reproductive material and the bone marrow are used for both therapeutic and research purposes.

Consequently, the following is an attempt to understand the concept of commercialization and whether it could act beneficially within certain contexts or contrariwise, cause great harm.
\end{abstract}

Keywords: commodification, property rights on the human body, monetary compensation for donors, law, society. 


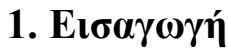

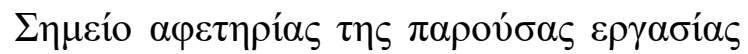
$\alpha \pi 0 \tau \varepsilon \lambda \varepsilon i ́$ to $\varepsilon \xi \dot{\eta} \varsigma \quad \varepsilon \rho \omega ́ \tau \eta \mu \alpha: \quad \pi \omega ́ \varsigma$ $\alpha v \tau \imath \lambda \alpha \mu \beta \alpha v o ́ \mu \alpha \sigma \tau \varepsilon \quad \tau \eta v \quad \varepsilon ́ v v 01 \alpha \quad \tau \eta \varsigma$

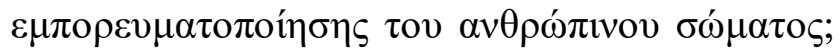

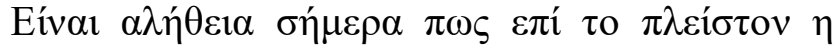

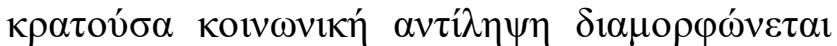

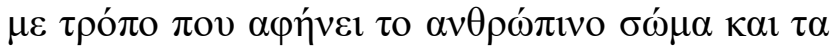

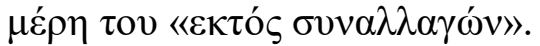

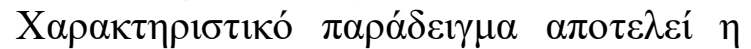

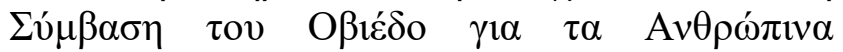

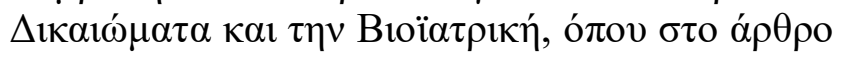

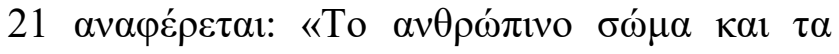

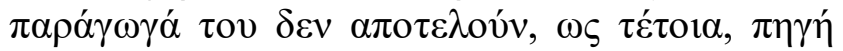

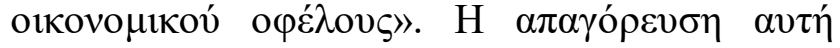

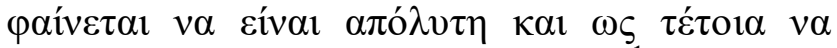

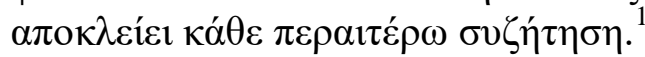

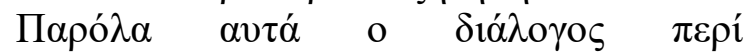

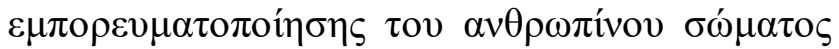

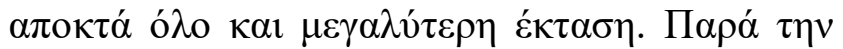

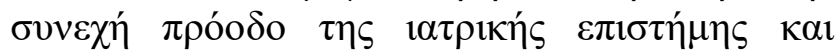

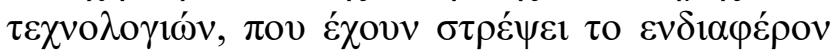

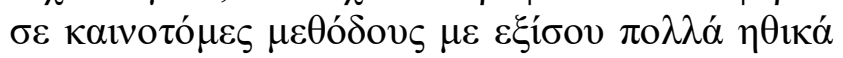

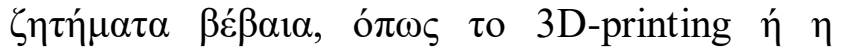

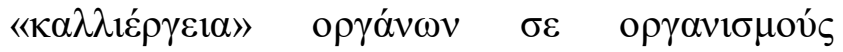

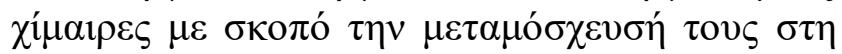

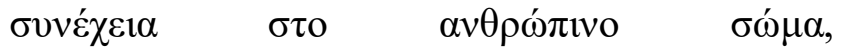

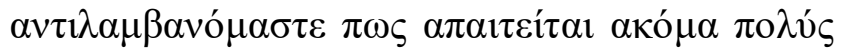

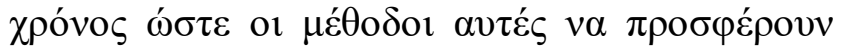

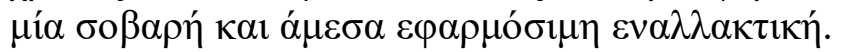

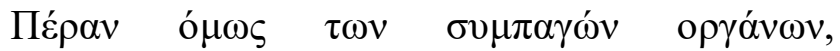

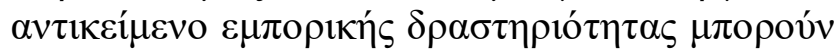

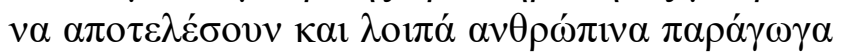

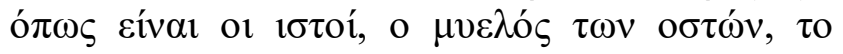

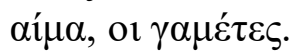

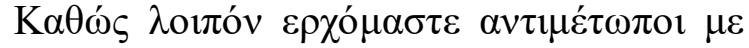

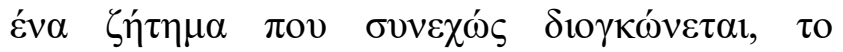

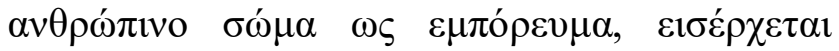

\footnotetext{
${ }^{1} \Delta \iota \alpha \theta \dot{\sigma} \sigma \iota \mu \sigma \tau о$

https://rm.coe.int/CoERMPublicCommonSearchServices/ DisplayDCTMContent?documentId=090000168007 $\mathrm{cf9} 9$
}

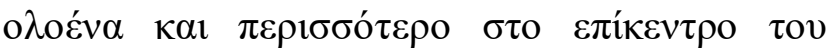
$\varepsilon v \delta 1 \alpha \varphi \varepsilon ́ \rho o v \tau o \zeta$.

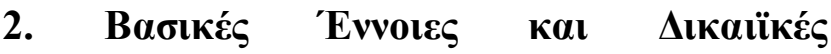

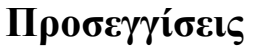

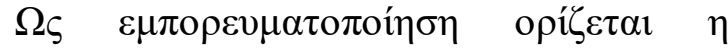

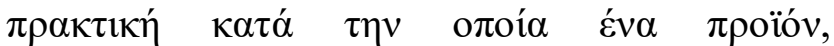

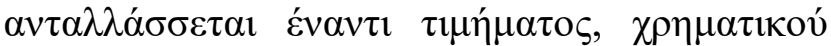

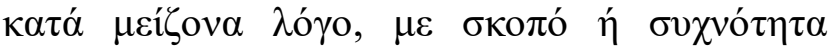

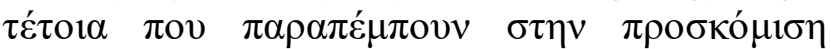

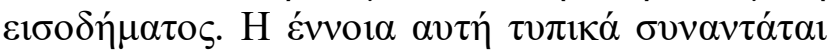

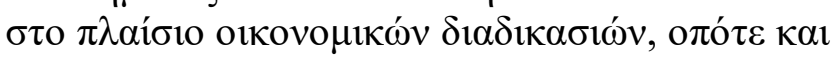

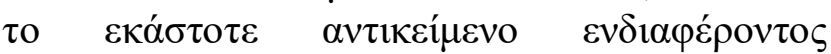

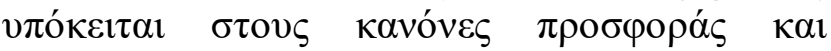

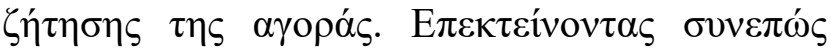

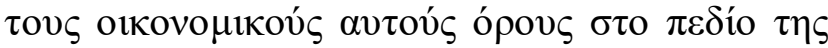

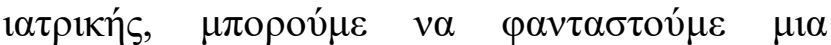

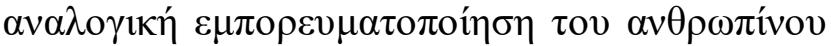
$\sigma \omega ́ \mu \alpha \tau o s$.

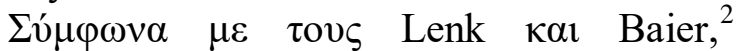

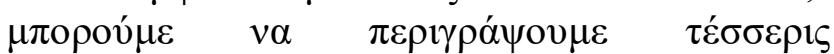

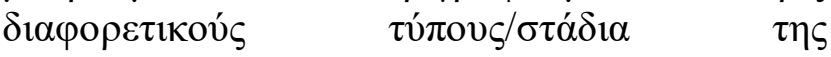

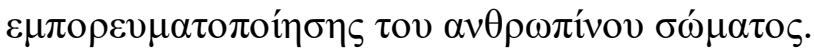

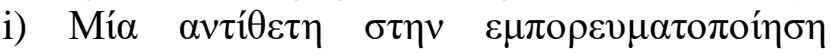

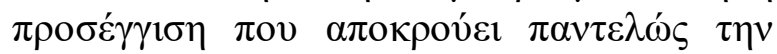

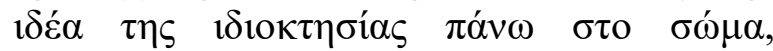

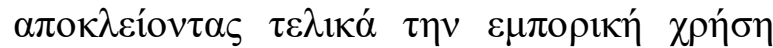

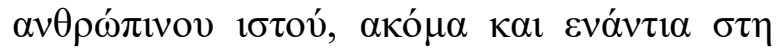

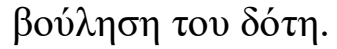

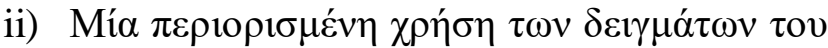

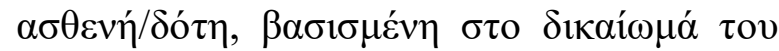

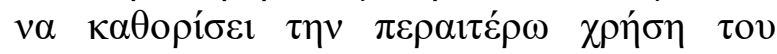

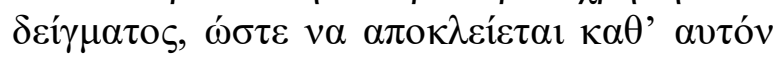

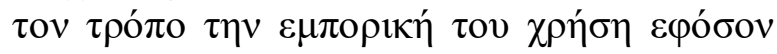
$\alpha v \tau o ́$ $\varepsilon \pi \imath \theta v \mu \varepsilon i ́$.

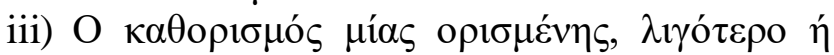

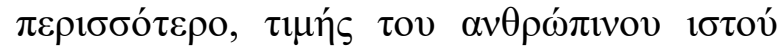

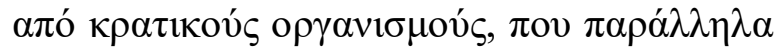

\footnotetext{
${ }^{2}$ Lenk C, Beier K. Is the commercialisation of human tissue and body material forbidden in the countries of the European Union?. J Med Ethics 2012,38:342-346.
} 


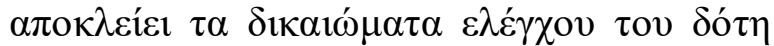

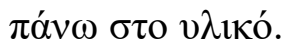

iv) $\mathrm{H} \quad \pi \lambda \eta \dot{\eta} \rho \varsigma_{\varsigma} \quad \varepsilon \mu \pi \circ \rho \varepsilon v \mu \alpha \tau$ o

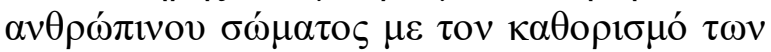

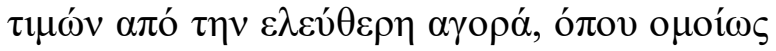

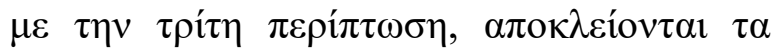

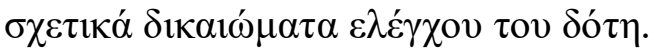

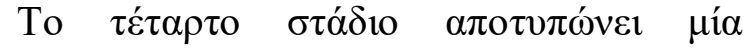

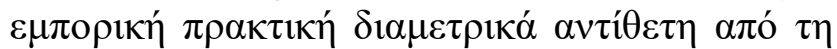

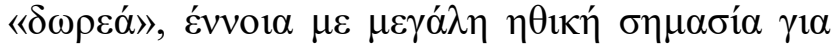

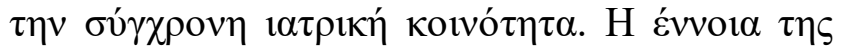

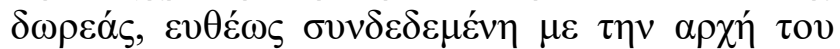

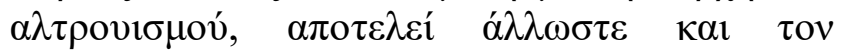

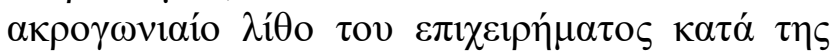

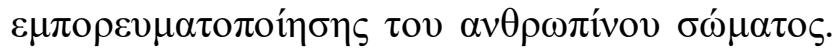

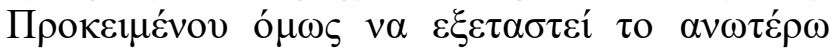

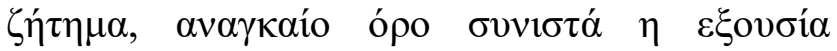

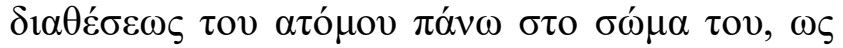

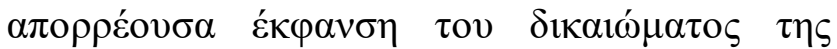

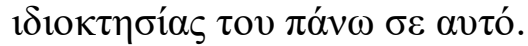

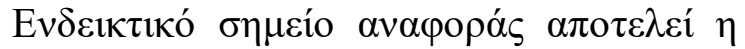

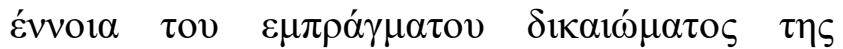

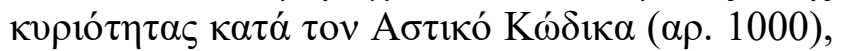

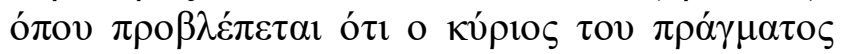

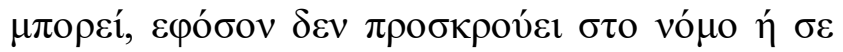

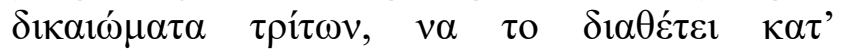

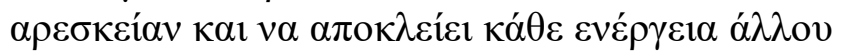

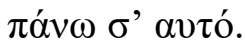

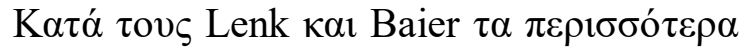

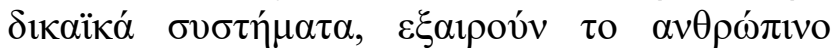

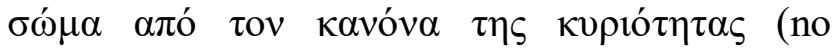

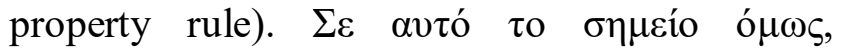

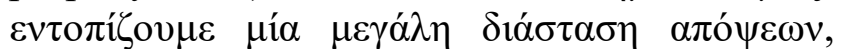

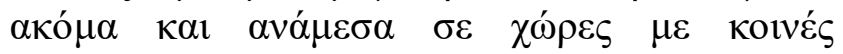

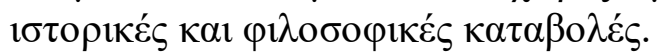

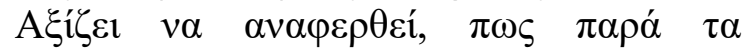

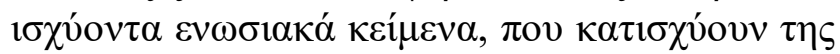

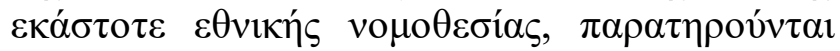

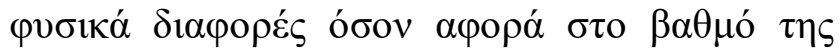

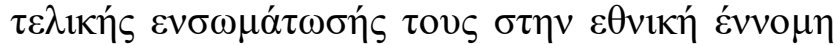

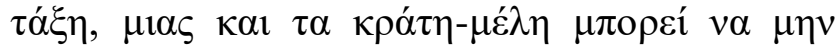

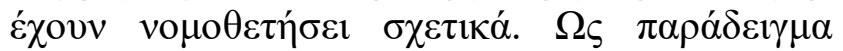

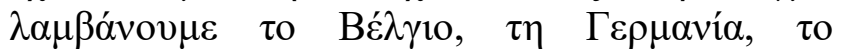

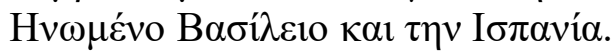

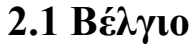

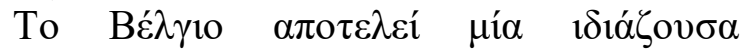

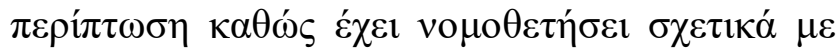

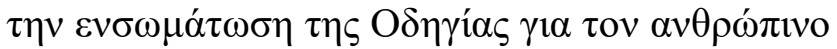

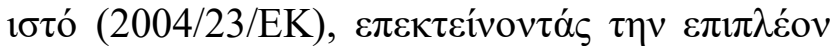

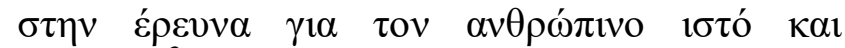

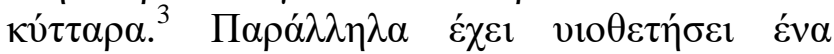

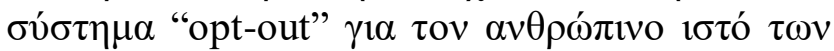

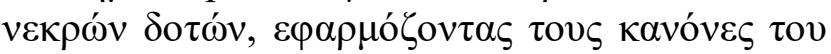

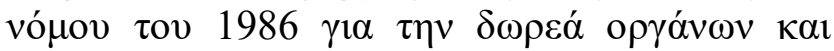

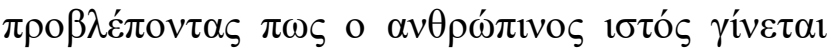

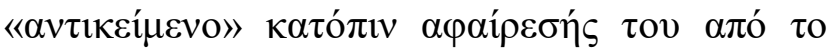

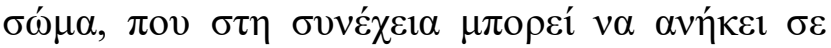

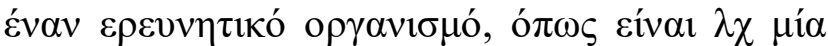

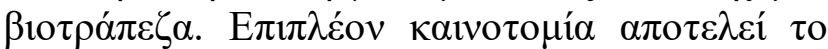

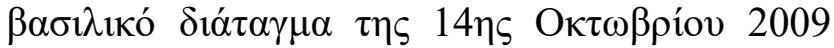

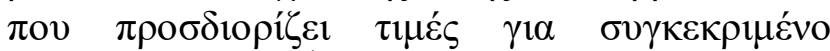

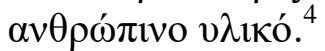

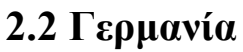

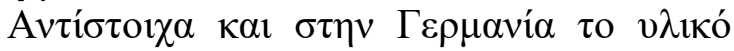

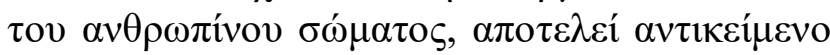

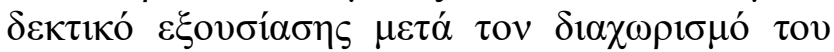

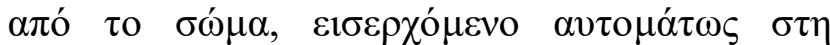

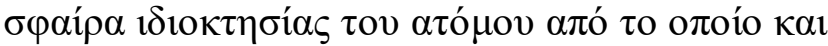

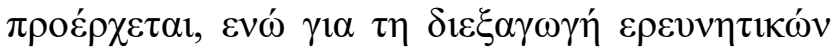

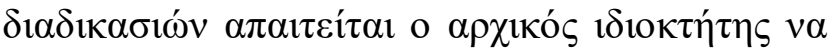

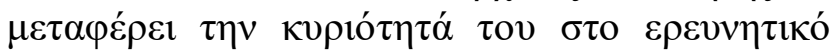

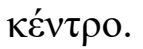

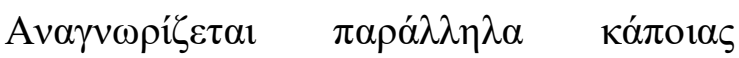

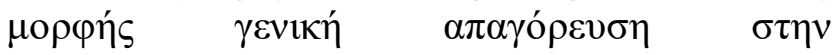

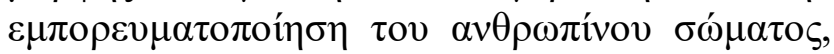

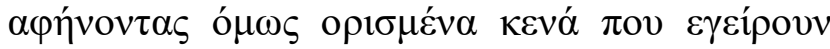

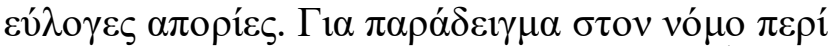
$\mu \varepsilon \tau \alpha \mu о \sigma \chi \varepsilon v ́ \sigma \varepsilon \omega \nu \quad$ (Transplatationsgesetz) ${ }^{5} \quad \sigma \tau \mathrm{o}$

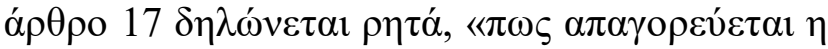

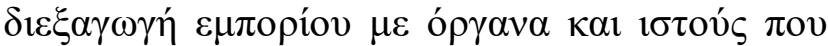

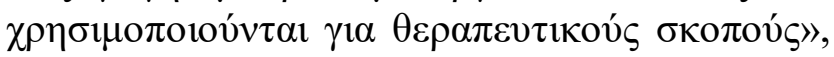

\footnotetext{
${ }^{3}$ Loi relative a l'obtention et a l'utilisation de materiel corporel humain destine a des applications medicales humaines ou a des fins de recherch scientifique. $\Delta \iota \alpha \theta \varepsilon ́ \sigma \iota \mu o$ $\sigma \tau o$

http://www.ejustice.just.fgov.be/cgi_loi/change_lg.pl?lang uage $=$ fr \&la $=F \& c n=2009092807 \&$ table $\_$name $=$loi

${ }^{4}$ Pirnay JP, Vanderkelen A, Zizi M, et al. Human cells and tissues: the need for a global ethical framework. Bull WHO 2010, 88:871.

$51 \alpha \theta \dot{\varepsilon} \sigma l \mu o \quad \sigma \tau o \quad$ https://www.gesetze-im-
internet.de/tpg/TPG.pdf
} 


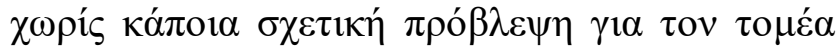

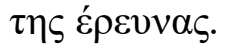

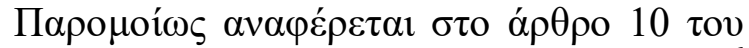
vó $\mu$ ov $\pi \varepsilon \rho i ́ ~ \mu \varepsilon \tau \alpha \gamma \gamma i ́ \sigma \varepsilon \omega v$ (Transfusionsgesetz) ${ }^{6}$

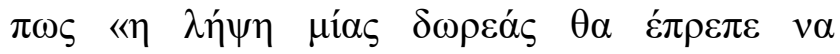

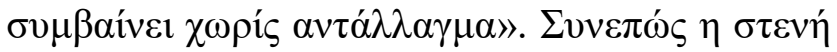

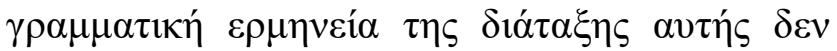

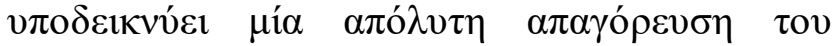

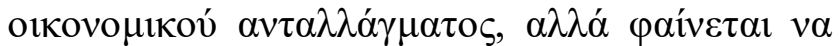

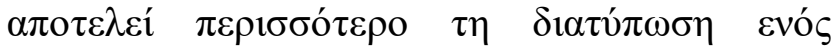

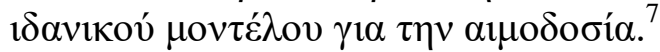

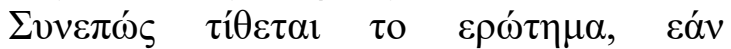

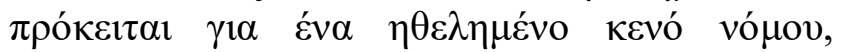

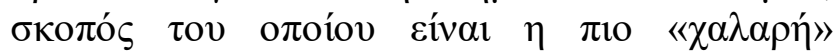

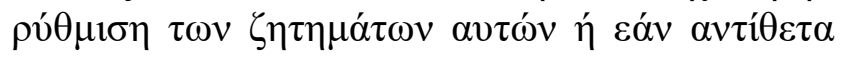

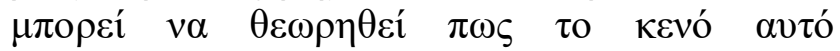

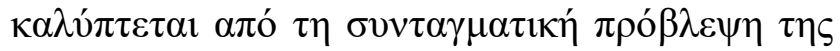

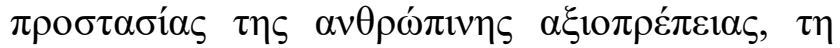

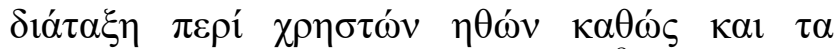

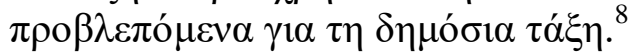

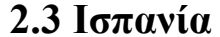

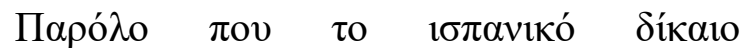

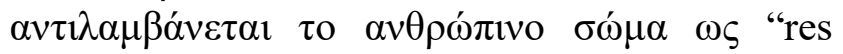

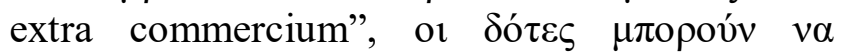

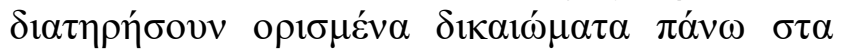

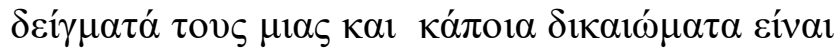
$\sigma v v \delta \varepsilon \delta \varepsilon \mu \varepsilon \dot{\varepsilon} v \alpha \quad \mu \varepsilon \quad \tau \eta v \quad \pi \rho 0 \sigma \tau \alpha \sigma i ́ \alpha \quad \tau \eta \varsigma$

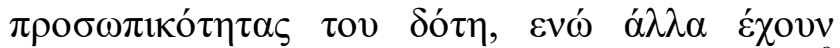

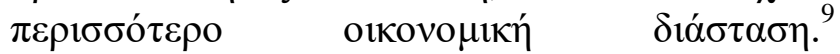

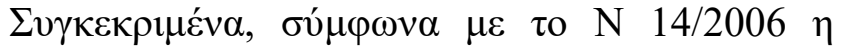

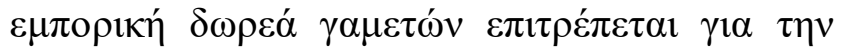

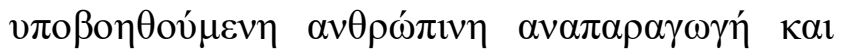

\footnotetext{
${ }^{6} \Delta \iota \alpha \theta \dot{\varepsilon} \sigma \iota \mu \sigma \tau о$

https://www.gesetze-im-internet.de/tfg/TFG.pdf

${ }^{7}$ Lenk C. Ökonomie der Körperteile: Wie weit reicht das Kommerzialisierungsverbot des menschlichen Körpers?. Berliner Debatte Initial 2010,21:9-18.

8 Taubnitz J. Das Verbot der Kommerzialisierung des menschlichen Körpers und seiner Teile. Kommerzialisierung des menschlichen Körpers. Berlin, Heidelberg: Springer, 2007:4.

${ }^{9}$ Report of the 3rd International Workshop of the Tiss.Eu Paris: EU Project, 2009. $\Delta \iota \alpha \theta \varepsilon \dot{\sigma} \sigma \mu о \quad \sigma \tau o$ http://www.tisseu.uni-

hannover.de/images/files/report_paris_final.pdf
}

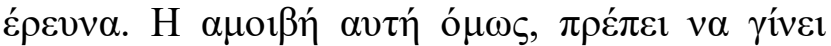

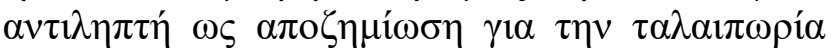

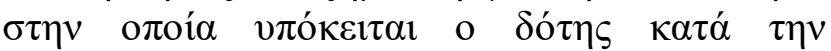

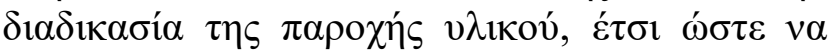

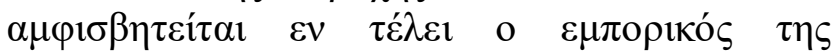
$\chi \alpha \rho \alpha \kappa \tau \eta ́ \rho \alpha \varsigma$.

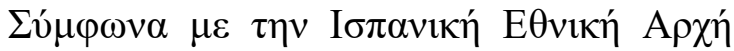

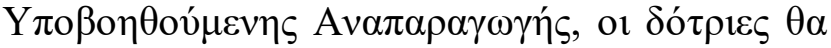
$\varepsilon \dot{\pi} \rho \varepsilon \pi \varepsilon \quad v \alpha \quad \lambda \alpha \mu \beta \alpha ́ v o v v \quad \omega \varsigma \quad \alpha \pi \circ \zeta \eta \mu i ́ \omega \sigma \eta$,

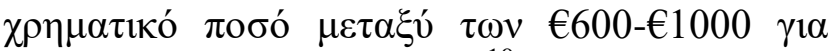

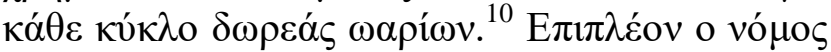

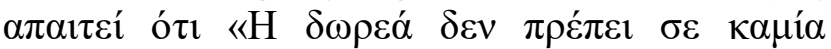

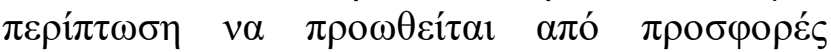

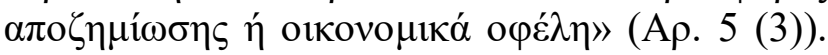

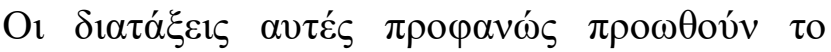

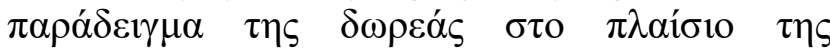

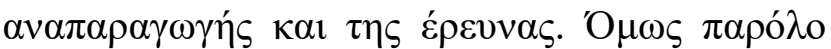

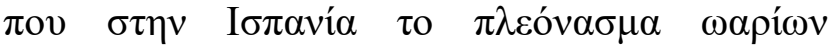

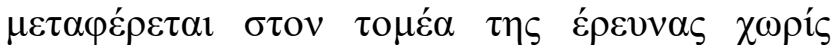

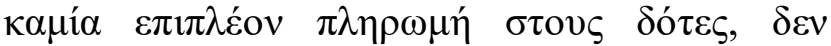

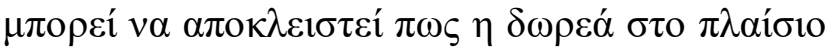

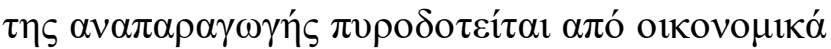

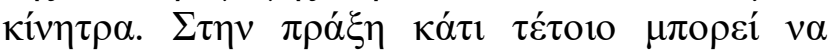

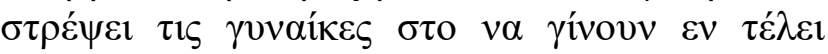

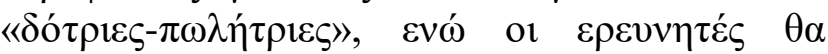

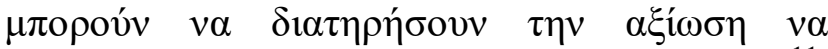

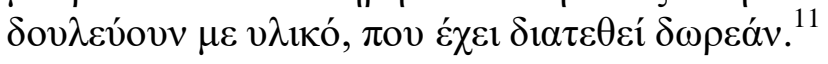

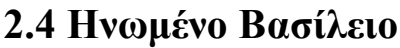

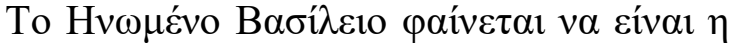

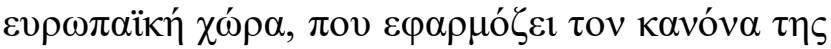

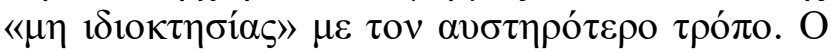

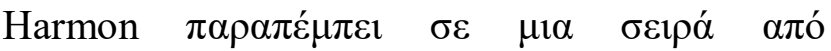

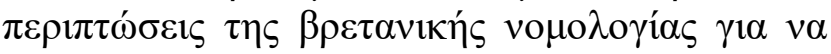

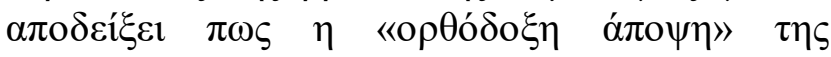

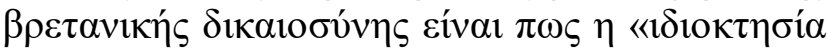

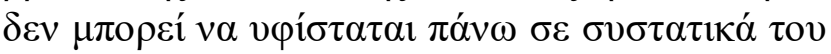

\footnotetext{
${ }^{10}$ Velasco JG. Egg donation in Spain. The Spanish point of view. Focus on Reproduction. Newsletter of the European Society of Human Reproduction and embryology, 2007,5:26-30.

11 Schultz S, Braun K. Spendende Verkäuferinnen. Eizellen für die Klonforschung. Berliner Debatte Initial 2010:21:28-40.
} 


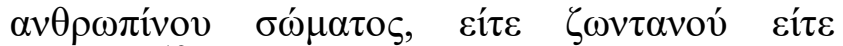
vยкрои́». ${ }^{12}$

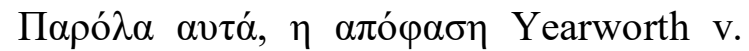

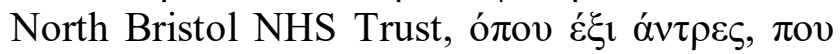

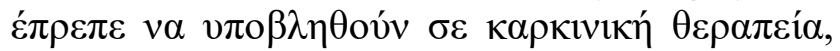

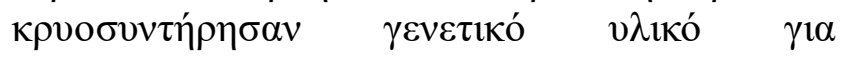

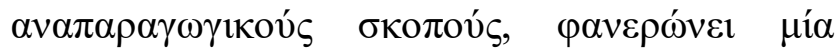

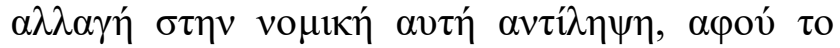

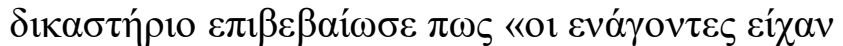

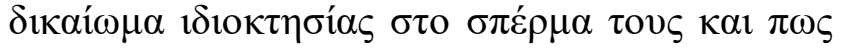

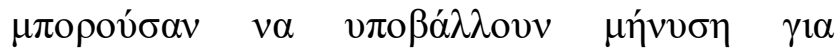

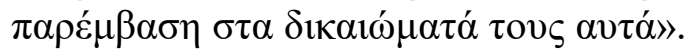

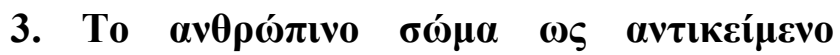

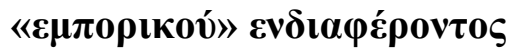

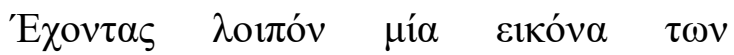

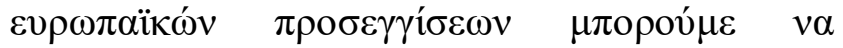

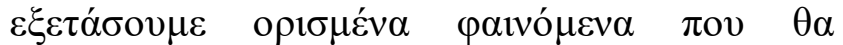

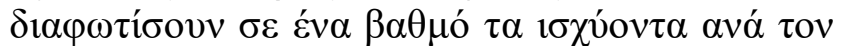

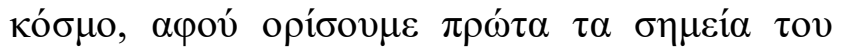

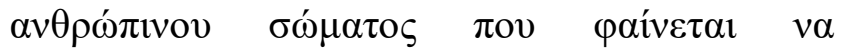

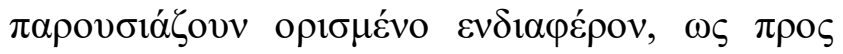

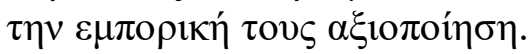

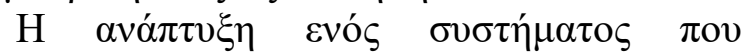

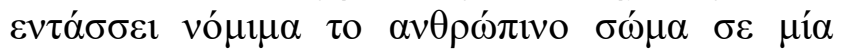

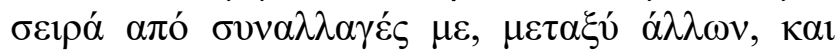

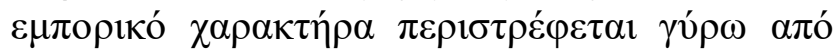

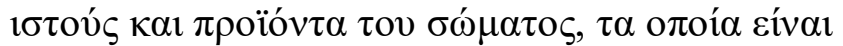

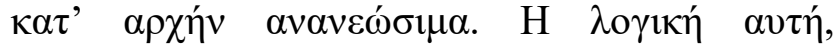

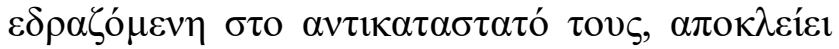

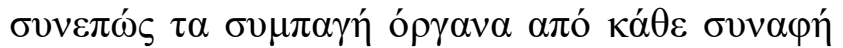

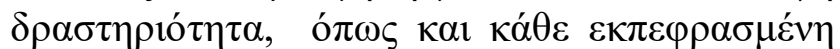

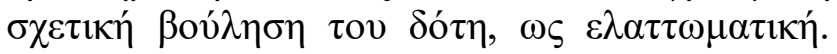

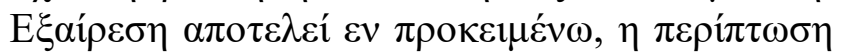

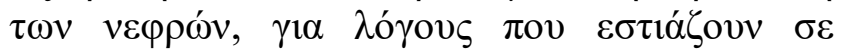

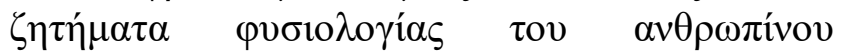

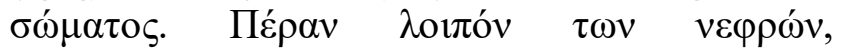

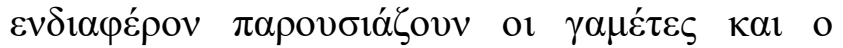

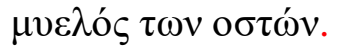

\footnotetext{
${ }^{12}$ Harmon SH. Yearworth v. North Bristol NHS Trust: a property case of uncertain significance?. Med Health Care Philos 2010,13:343-350.
}

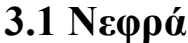

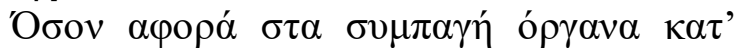
$\varepsilon \xi o \chi \eta ́ v \quad \pi \varepsilon \rho i ́ \pi \tau \omega \sigma \eta, \quad \mu \varepsilon \quad \sigma \omega \rho \varepsilon i ́ \alpha \quad \eta \theta 1 \kappa \omega ́ v$

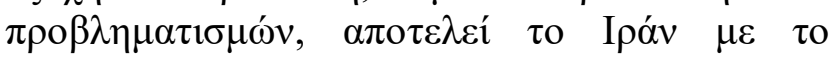

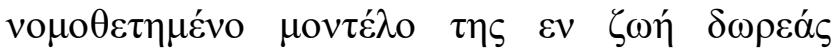

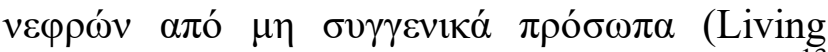
Unrelated Renal Donor $\varepsilon \varphi \varepsilon \xi \eta \dot{s}$ LURD). ${ }^{13}$

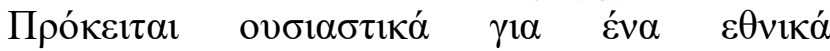

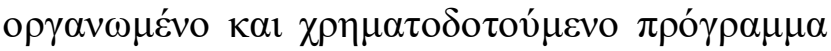

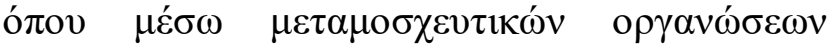

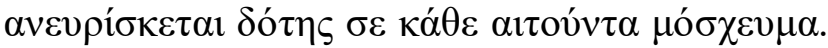

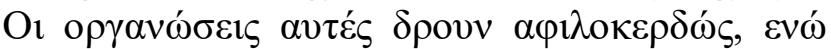

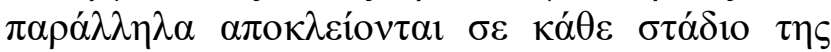

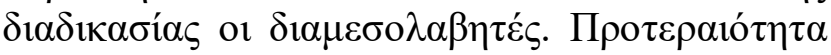

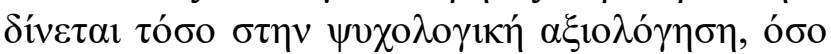

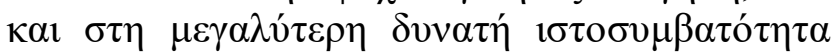

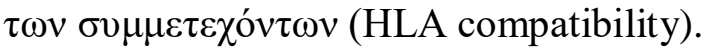

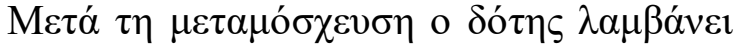

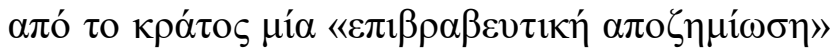

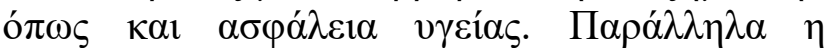

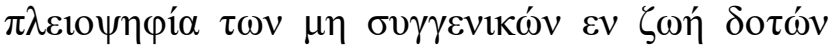

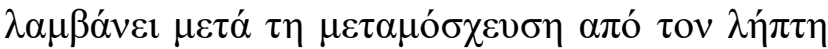

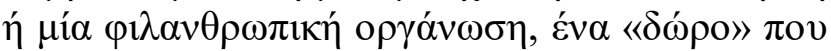

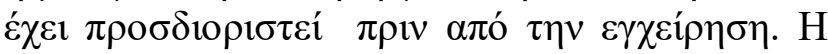

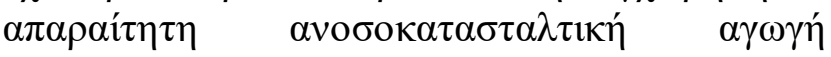

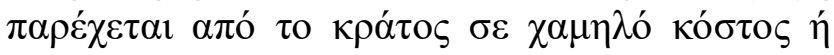

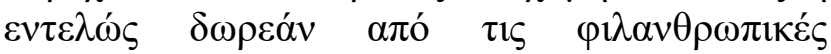

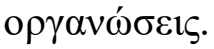

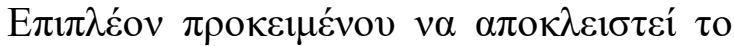

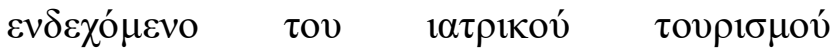

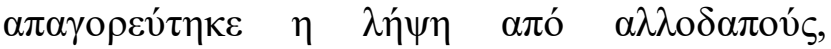

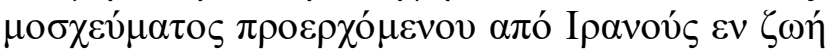

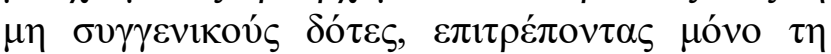

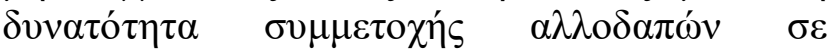
$\mu \varepsilon \tau \alpha \mu о \sigma \chi \varepsilon v ́ \sigma \varepsilon 1 \varsigma$ ó

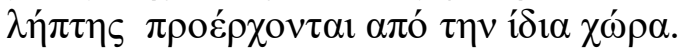

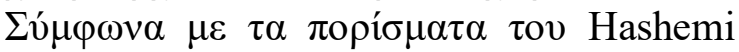

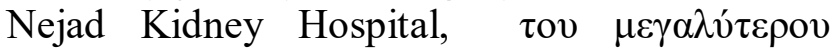

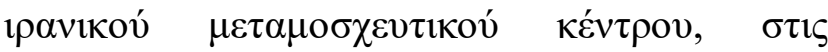

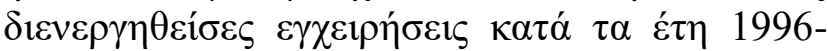

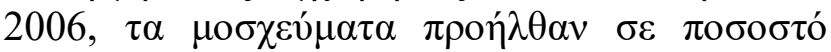

13 Ghods AJ, Savaj S . Iranian Model of Paid and Regulated Living-Unrelated Kidney Donation. Clin J Am Soc Nephrol 1 2006:1136-1145. 


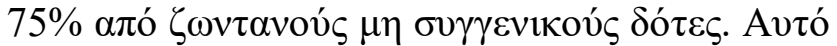

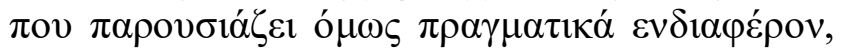

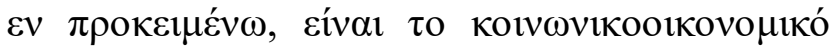

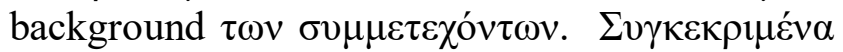

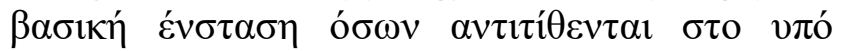

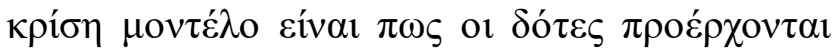

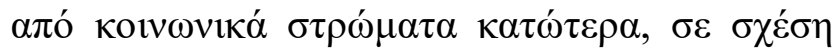

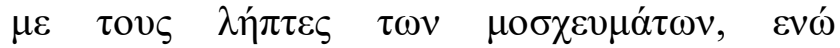

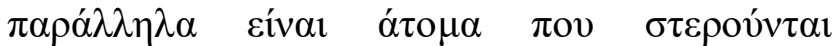

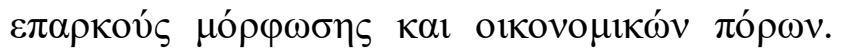

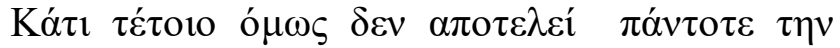
$\pi \varepsilon \rho i ́ \pi \tau \omega \sigma \eta$.

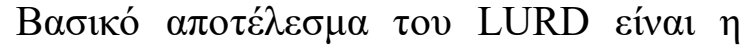

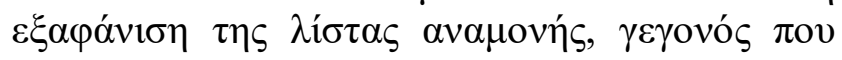

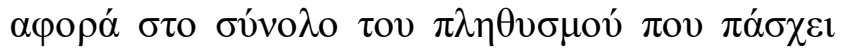

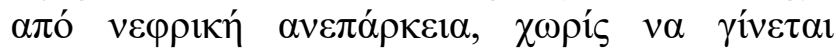

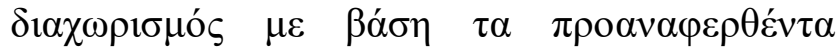

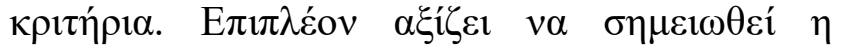

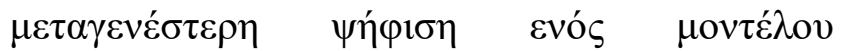

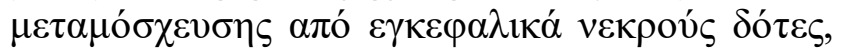

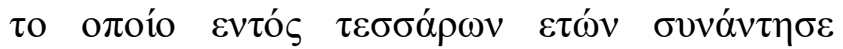

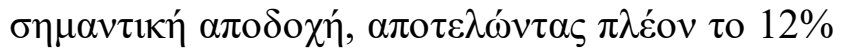

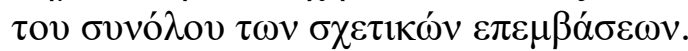

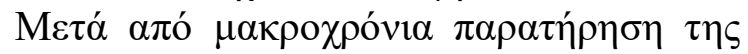

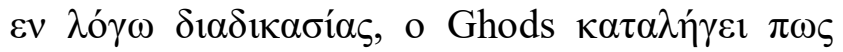

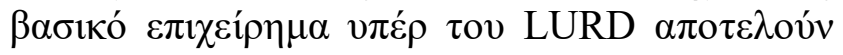

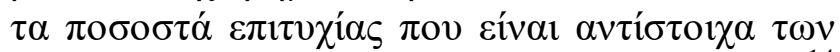

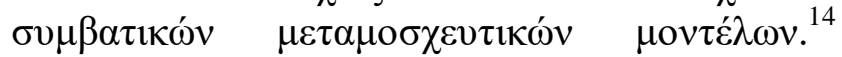

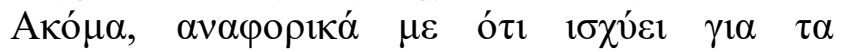

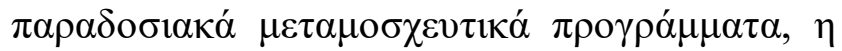

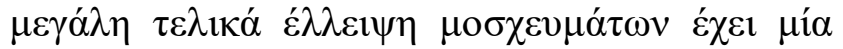

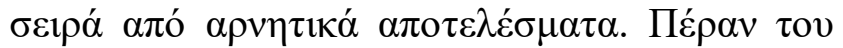

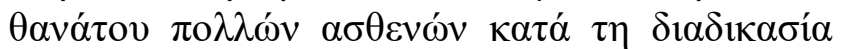

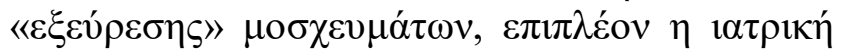

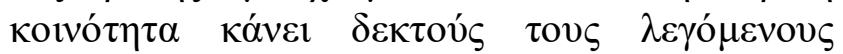

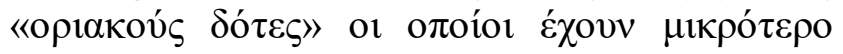

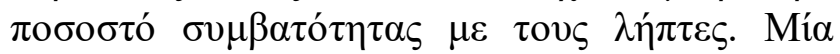

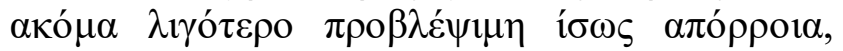

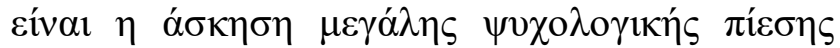

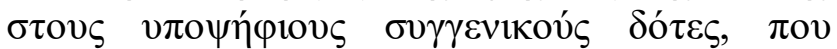

14 Voiculescu A, Ivens A. Kidney transplantation from related and unrelated living donors in a single German centre. Nephrology Dialysis Transplantation. 2003,18(2):418-425.

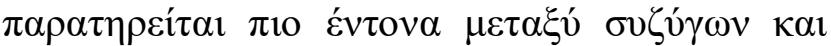

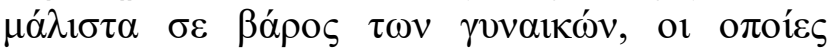

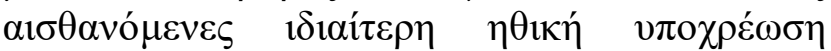

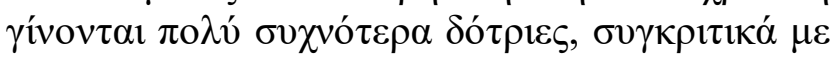
$\tau o v \varsigma \alpha ́ v \delta \rho \varepsilon \varsigma$.

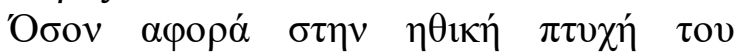

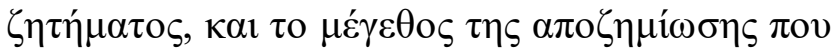

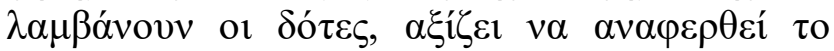

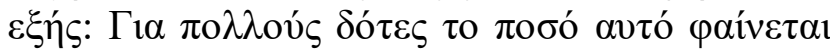

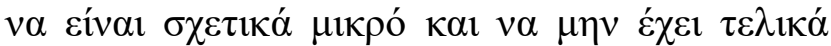

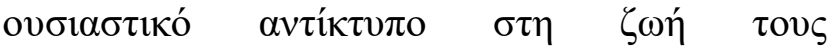

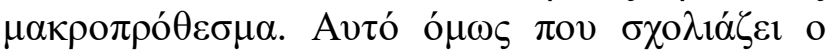

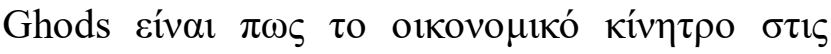

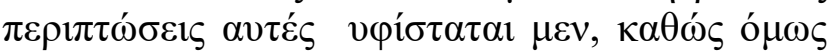

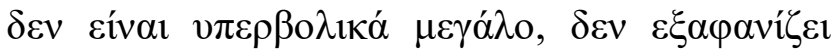

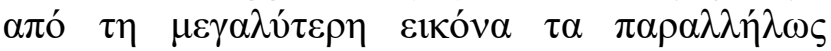

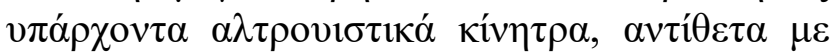

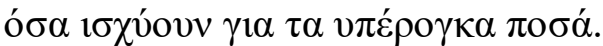

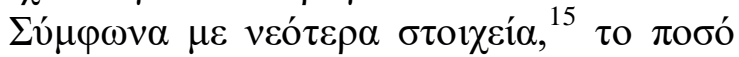

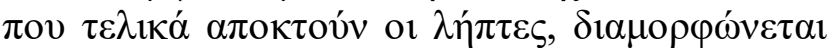

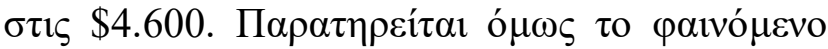

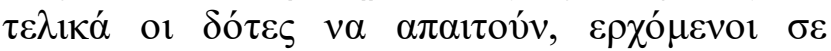

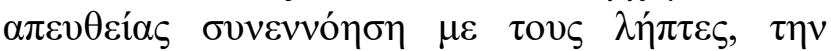

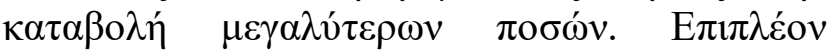

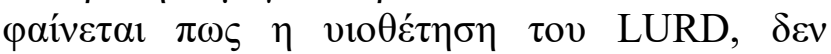

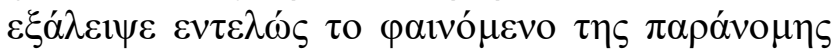

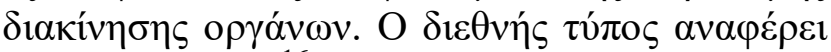

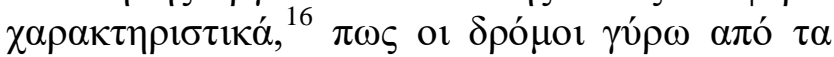

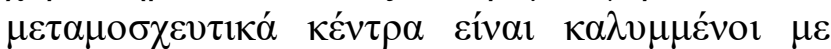

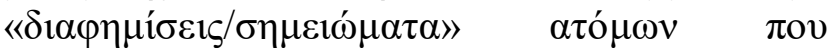

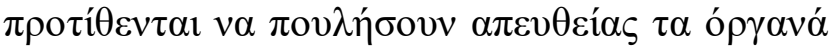

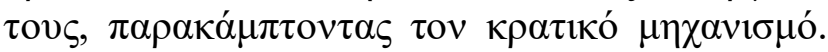

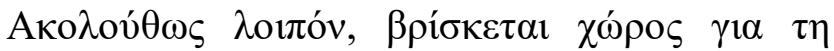
$\delta \rho \alpha ́ \sigma \eta \delta 1 \alpha \mu \varepsilon \sigma o \lambda \alpha \beta \eta \tau \omega ́ v$.

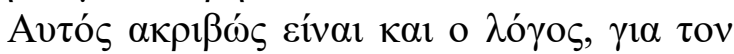

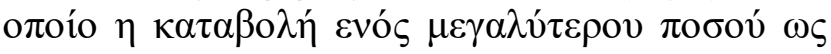
$\alpha \pi \circ \zeta \eta \mu i ́ \omega \sigma \eta$ a $\alpha \varepsilon v \theta \varepsilon i ́ \alpha \varsigma$ a

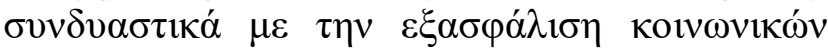

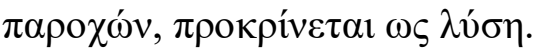

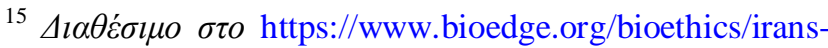
legal-market-in-kidneys-creates-a-black-market-too/12478

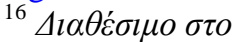

https://bpr.berkeley.edu/2018/05/03/legalizing-traffickingirans-unjust-organ-market-and-why-legal-selling-oforgans-should-not-be-the-resolve/ 


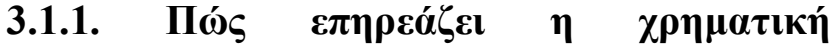

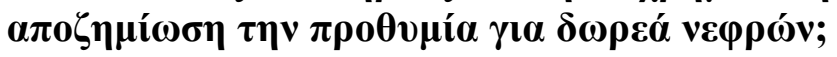

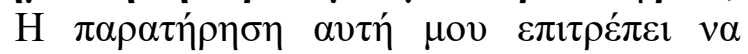

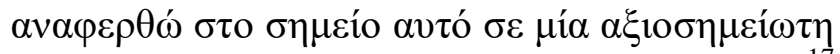

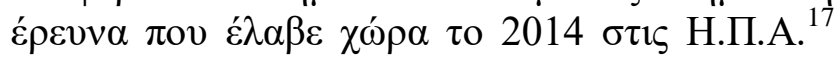

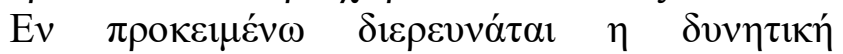

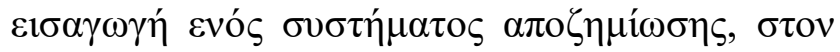

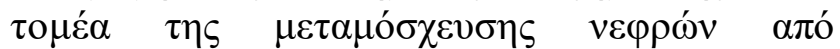

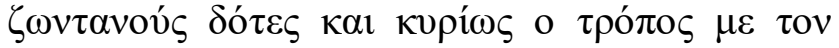

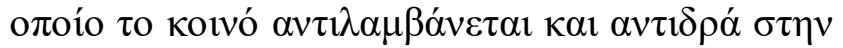

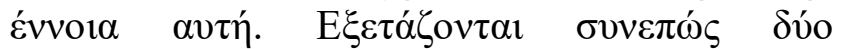

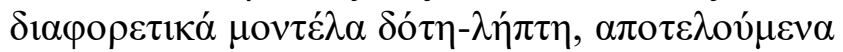

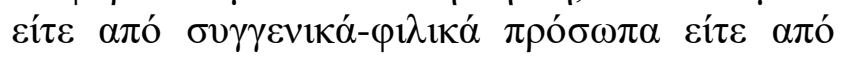

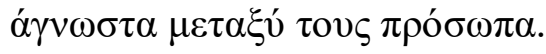

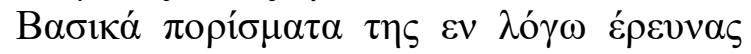
$\alpha \pi \circ \tau \varepsilon \lambda \circ v^{2} \tau \alpha \kappa \alpha ́ \tau \omega \theta$ :

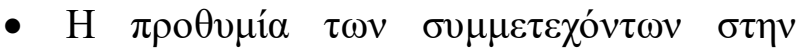

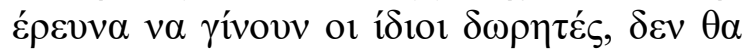

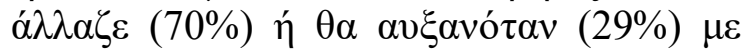
$\tau \eta \nu ~ \kappa \alpha \tau \alpha \beta о \lambda \eta \dot{~} \alpha \pi \circ \zeta \eta \mu i ́ \omega \sigma \eta s$.

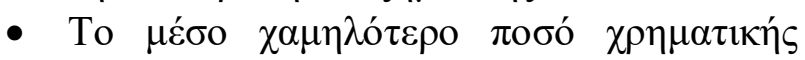

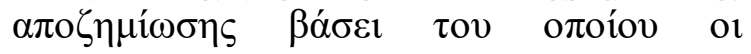

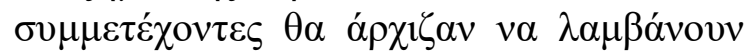

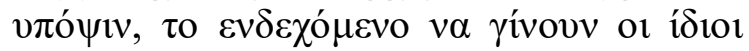

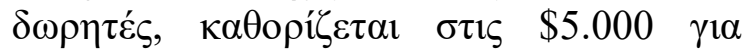

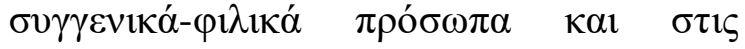

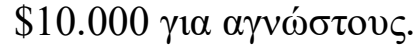

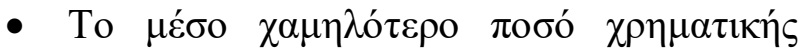

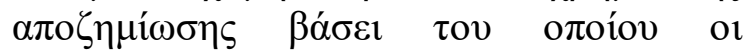

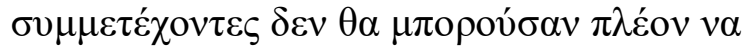
$\alpha \rho v \eta \theta$ oúv (undue inducement) va yívouv

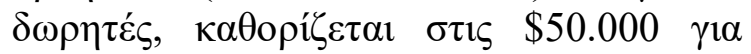

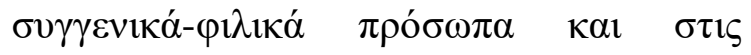

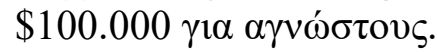

- Or $\pi \lambda \dot{\varepsilon}$ Ov $\pi \rho о \tau \mu \omega ́ \mu \varepsilon v \varepsilon \varsigma \quad \mu о \rho \varphi \varepsilon ́ \varsigma$

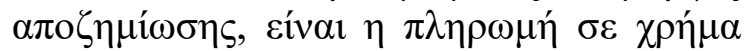

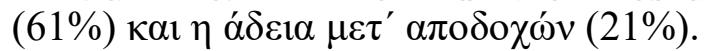

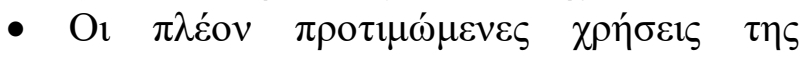

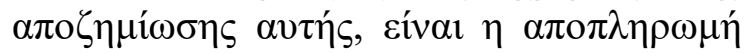

${ }^{17}$ Gordon EJ, Patel CH, Sohn M-W, Hippen B, Sherman A. Does Financial Compensation for Living Kidney Donation Change Willingness to Donate? American Journal of Transplantation 2015,15:265-273.

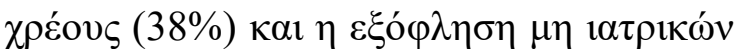

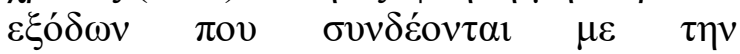
$\mu \varepsilon \tau \alpha \mu$ ó $\chi \varepsilon v \sigma \eta(29 \%)$.

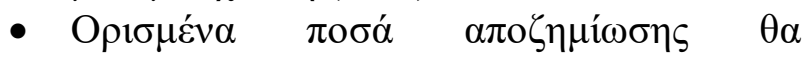

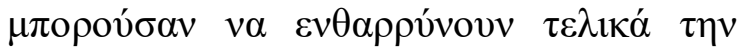

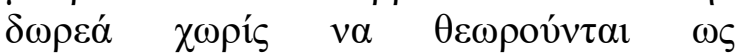

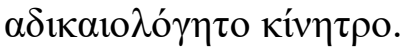

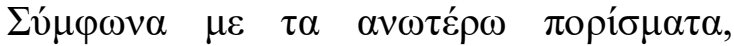

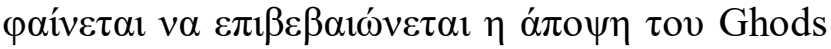

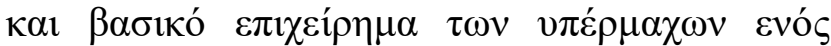

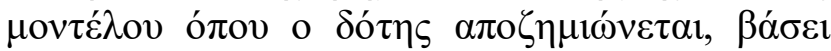

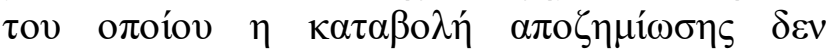

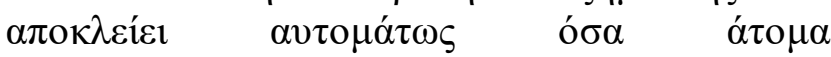

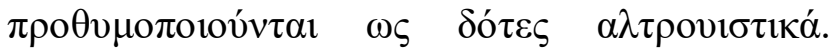
$\Sigma v \gamma \kappa \varepsilon \kappa \rho \mu \varepsilon \dot{v} \alpha \quad \gamma l \alpha \quad \tau \eta v \quad \pi \lambda \varepsilon 10 \psi \eta \varphi i ́ \alpha \quad \tau \omega v$

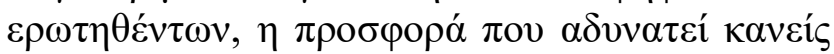

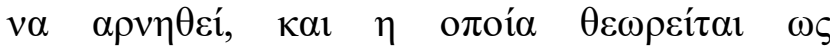
$\pi \alpha \rho \alpha \kappa_{i ́} \eta \sigma \eta, \quad \delta 1 \alpha \mu о \rho \varphi \omega ́ v \varepsilon \tau \alpha 1 \quad \sigma \varepsilon \quad \pi$

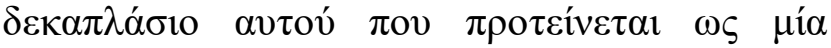
$\lambda о \gamma ı \kappa \eta ́ ~ \alpha \pi \circ \zeta \eta \mu i ́ \omega \sigma \eta . ~ \Sigma v v \varepsilon \pi \omega ́ \varsigma \tau \alpha ~ \sigma v \mu \pi \varepsilon \rho \alpha ́ \sigma \mu \alpha \tau \alpha$

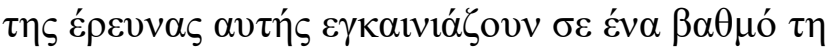

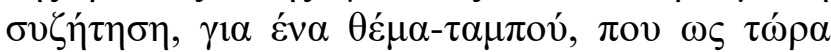

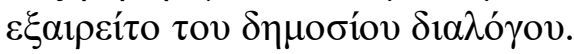

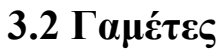

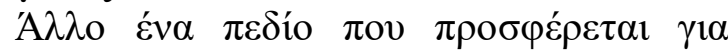

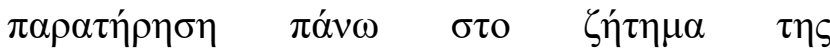

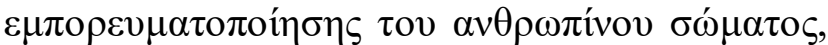

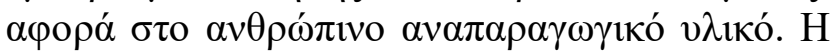

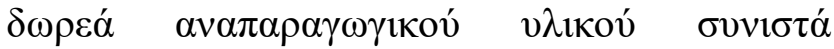

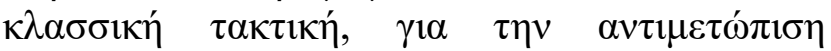

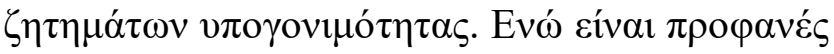

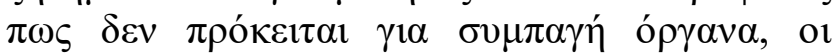

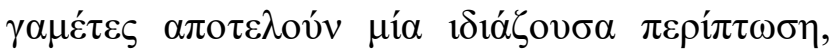

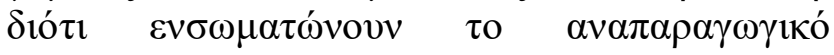

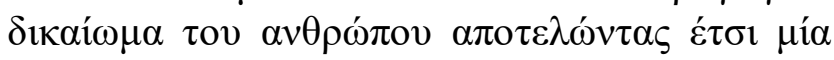

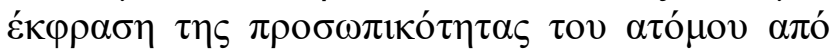

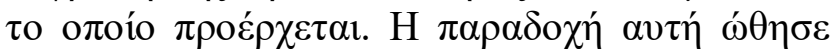

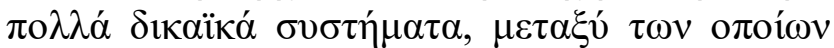

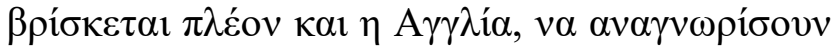

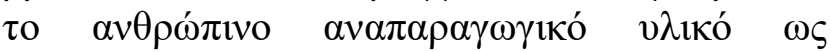

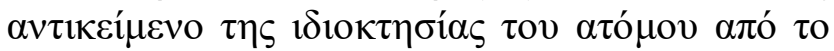
о тоío $\pi \rho \circ \varepsilon ́ \rho \chi \varepsilon \tau \alpha 1$. 


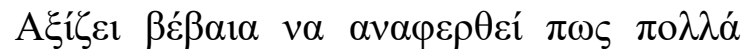

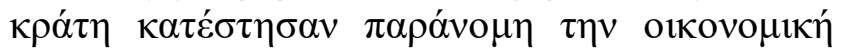

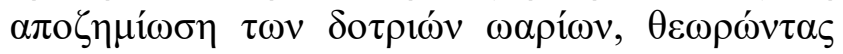

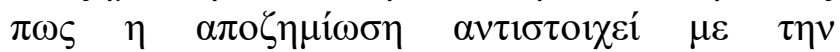

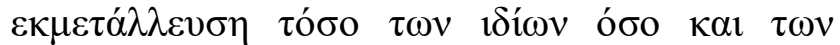

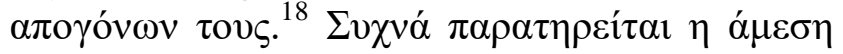

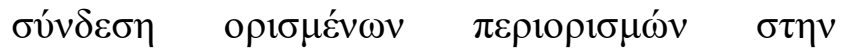

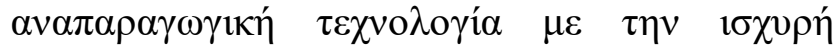

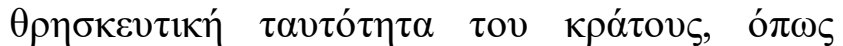

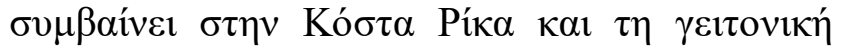

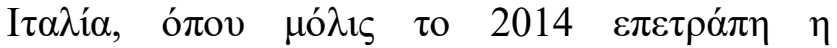

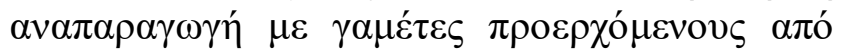

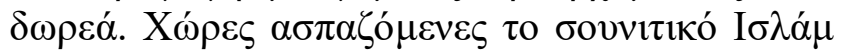

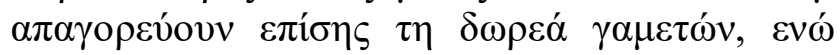

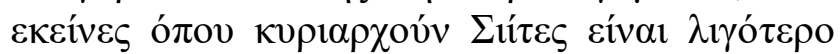

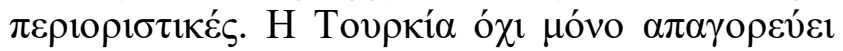

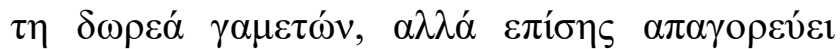

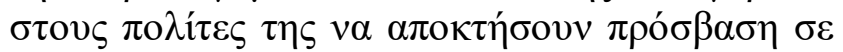

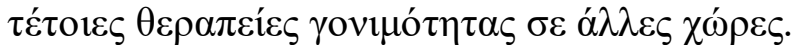

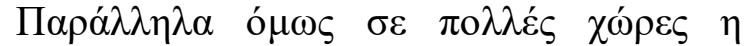

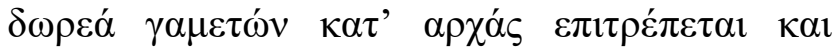
$\varepsilon \pi \imath \lambda \varepsilon \dot{\text { ov }} \quad \alpha \pi \circ \zeta \eta \mu \mathrm{i} v \varepsilon \tau \alpha 1 . \quad \Omega \varsigma \quad \alpha \pi \circ \zeta \eta \mu i ́ \omega \sigma \eta$

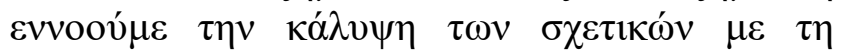

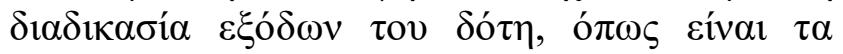

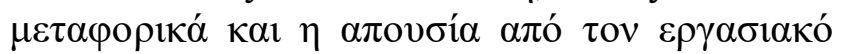

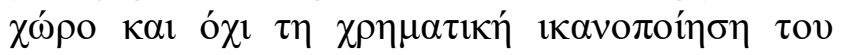

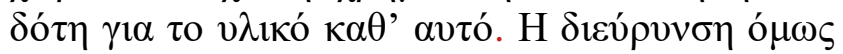

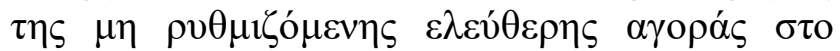

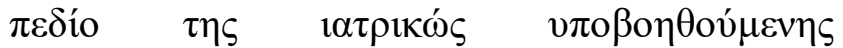

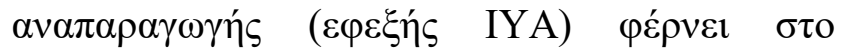

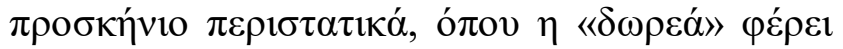

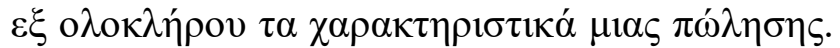

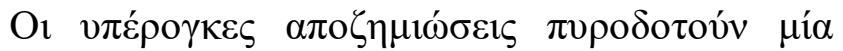

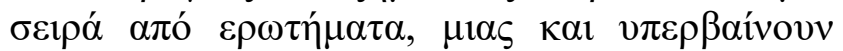

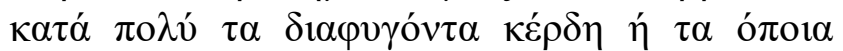

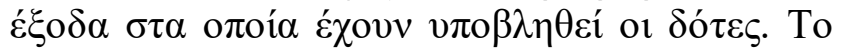

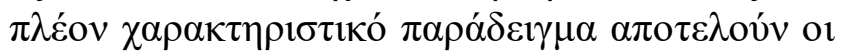
НПА.

\subsection{1 НПА}

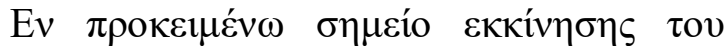

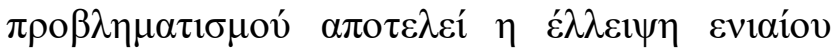

\footnotetext{
${ }^{18}$ Kenney N, McGowan M. Egg donation compensation: ethical and legal challenges. Dove Press, 2014,4:15-24.
}

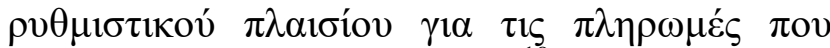

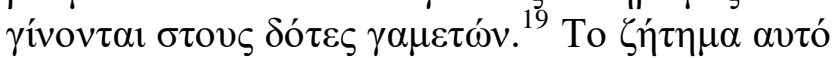

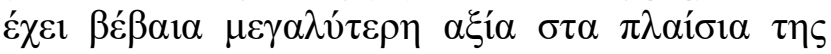

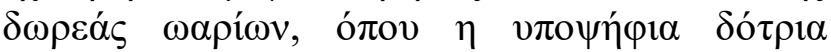

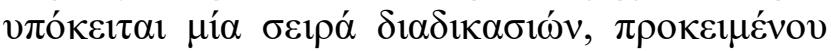

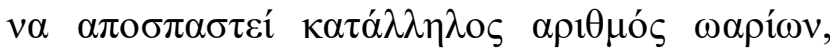

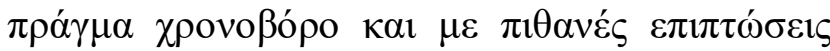

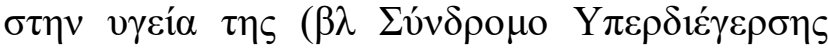

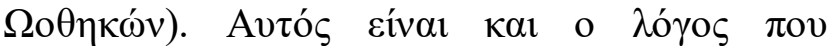

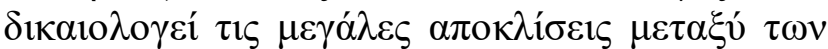

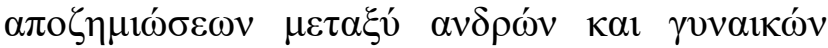

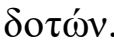

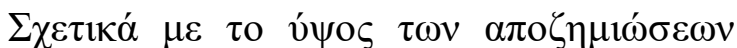

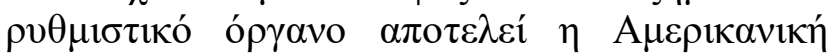

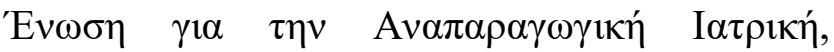

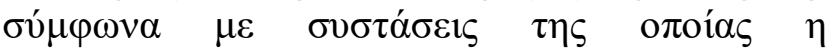

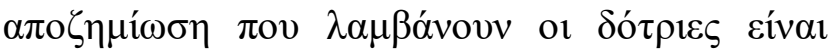

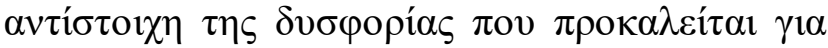

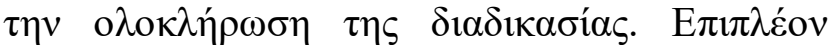

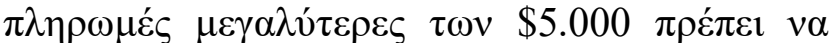

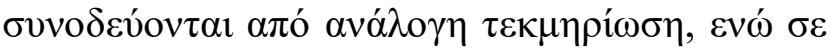

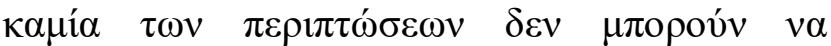

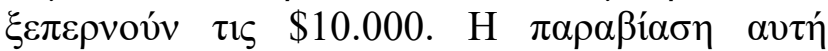

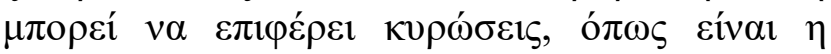

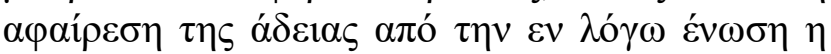

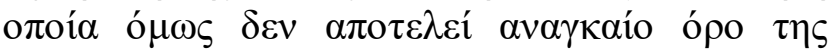

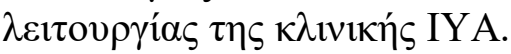

\subsubsection{1. $\Delta \omega \rho \varepsilon \alpha ́$ $\Sigma \pi \varepsilon ́ \rho \mu \alpha \tau 0 \varsigma$}

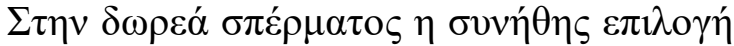

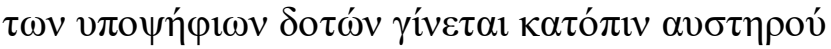

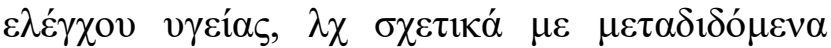

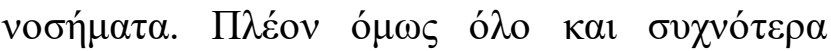

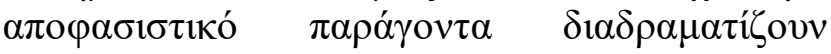

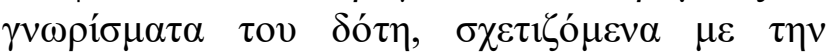

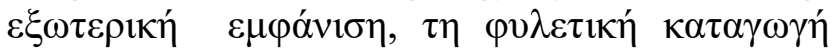

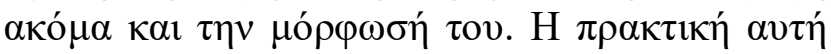

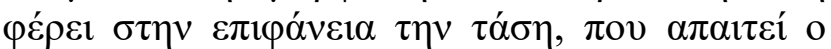

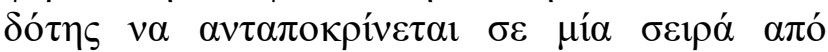

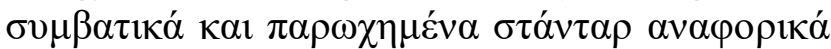

${ }^{19}$ Daniels C., Heidt-Forsythe E. Gendered Eugenics and the Problematic of Free Market Reproductive Technologies: Sperm and Egg Donation in the United States, Signs, 2012,37:719-747. 


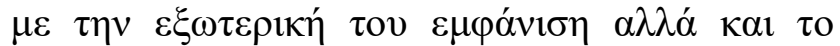

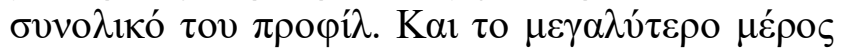

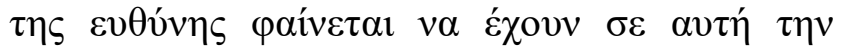

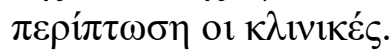

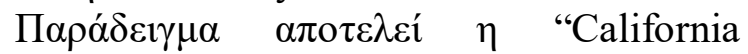

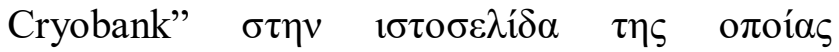

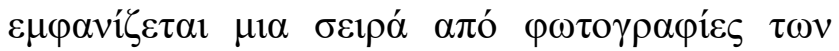

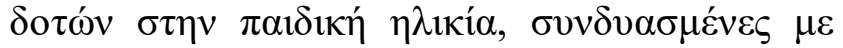

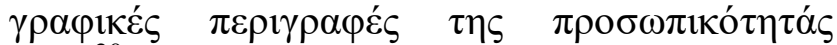

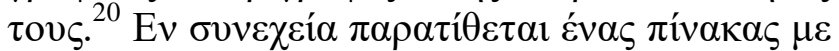

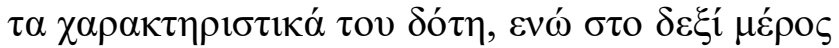

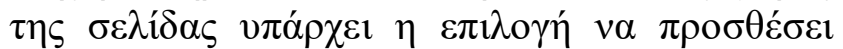

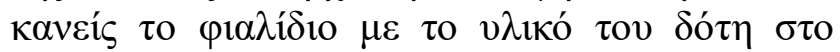

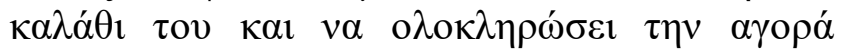

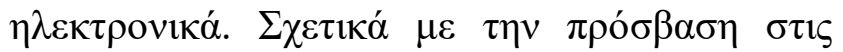

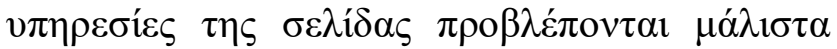

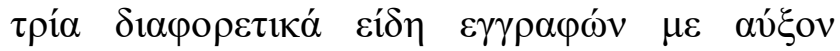

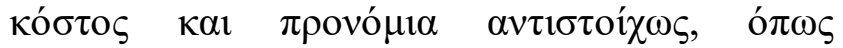

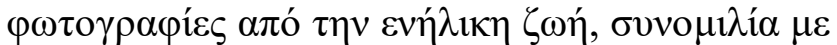

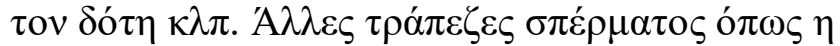

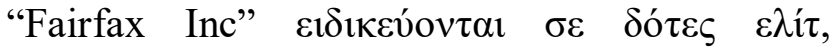

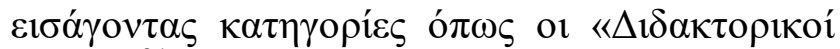
$\Delta$ ó $\varepsilon \varepsilon \zeta \gg .{ }^{21}$

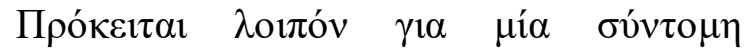

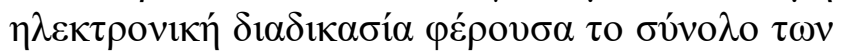

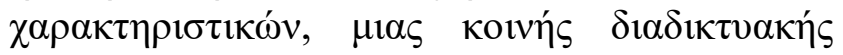

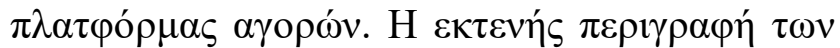

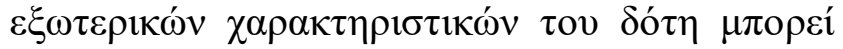

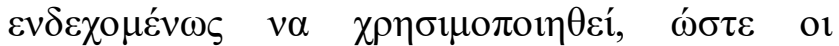

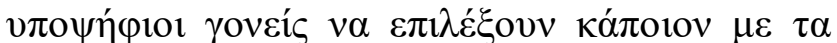

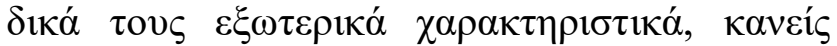

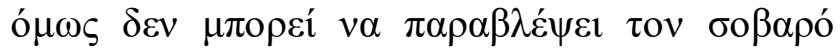

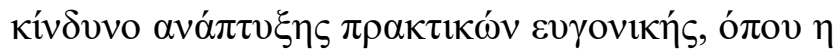

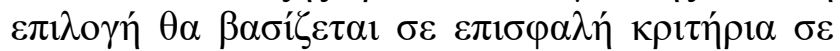

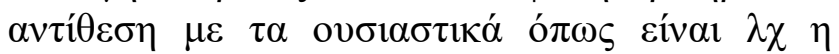

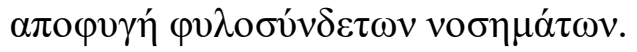

\subsubsection{2 $\Delta \omega \rho \varepsilon \alpha ́$ $\Omega \alpha \rho i ́ \omega v$}

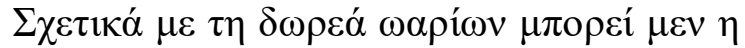

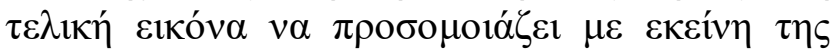

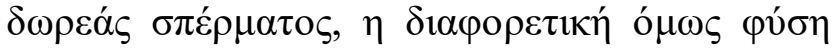

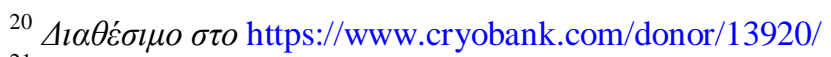

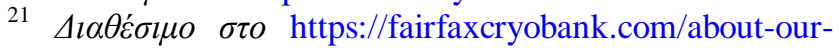
donors
}

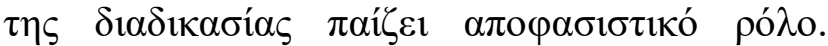

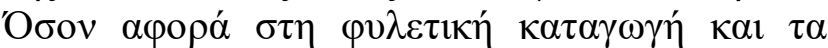

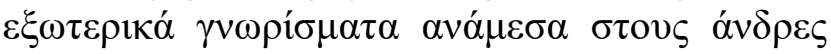

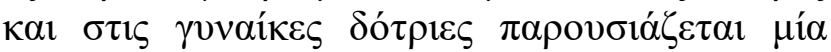

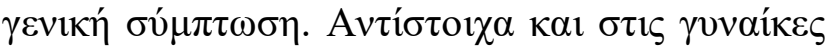

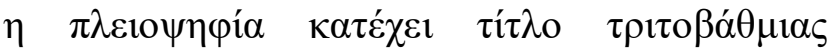

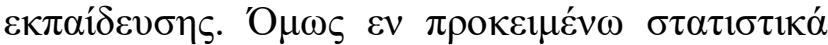

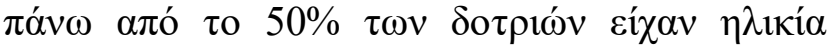

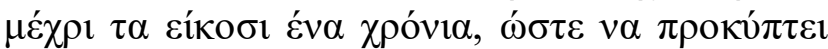

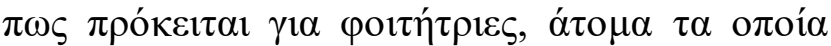

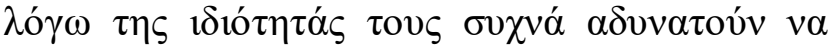

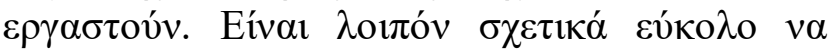

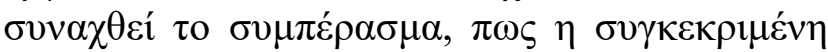

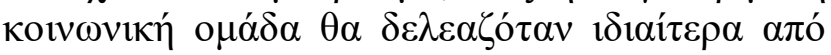

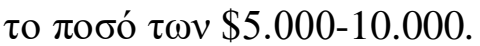

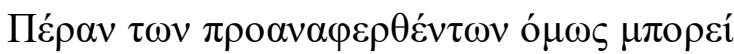

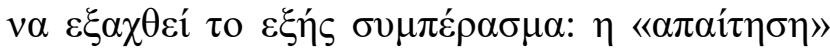

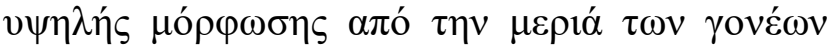

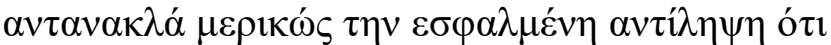

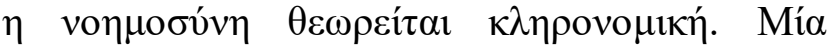

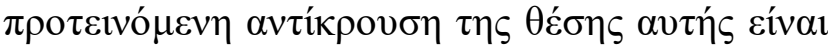

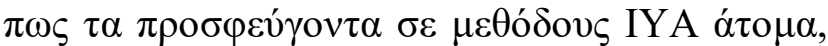

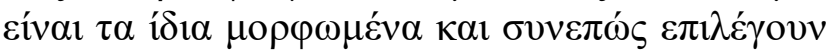

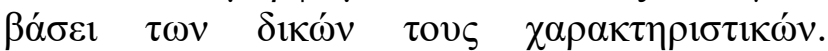

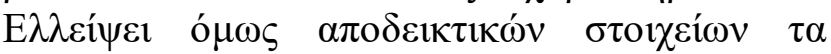

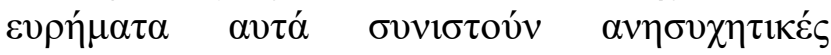
$\varepsilon v \delta \varepsilon i ́ \xi \varepsilon 1 \varsigma \mu 1 \alpha \varsigma$ $1 \varepsilon \rho \alpha ́ \rho \chi \eta \sigma \eta \varsigma ~ \tau \omega \nu ~ \pi \rho о \tau \imath \mu \omega ́ \mu \varepsilon v \omega \nu$

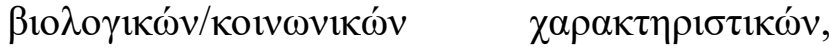

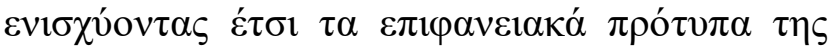

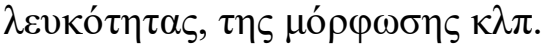

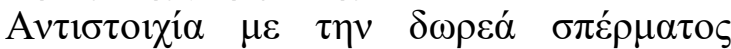

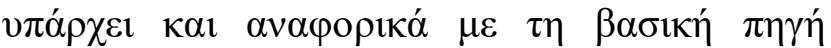

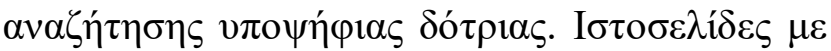

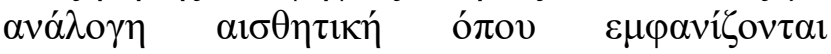

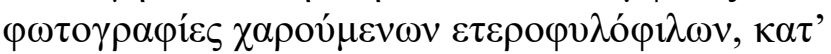

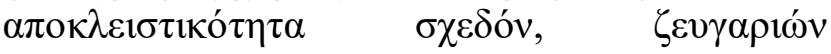

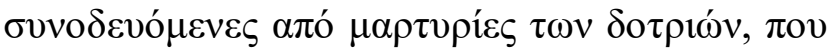

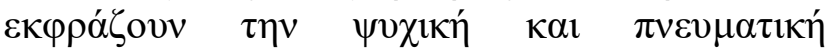

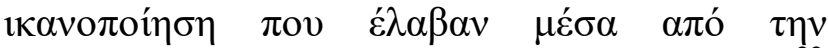

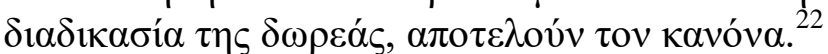

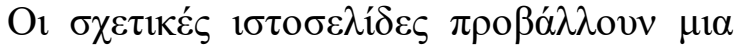

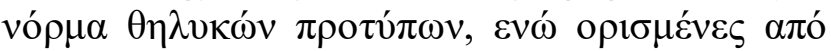

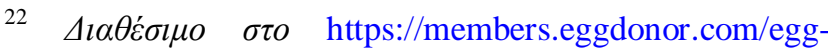
donor-database
} 


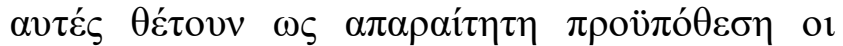

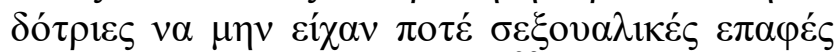

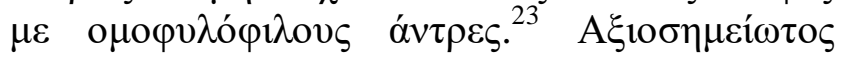

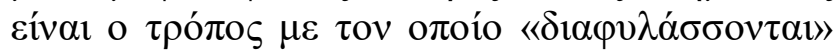

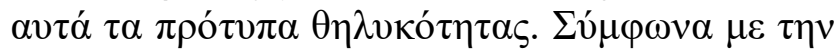
$1 \sigma \tau 0 \sigma \varepsilon \lambda i \delta \alpha \alpha \eta \varsigma$ "Egg Donation Inc" $« \mu 1 \alpha$

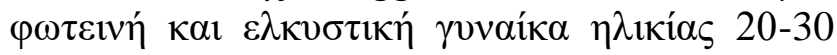

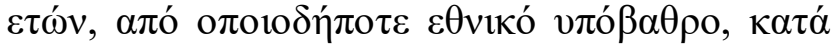

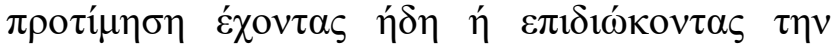

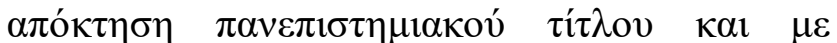

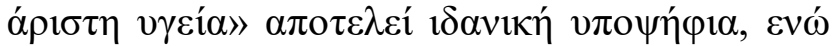

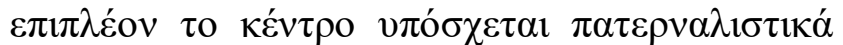

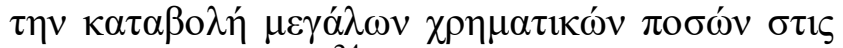

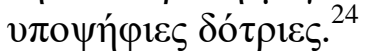

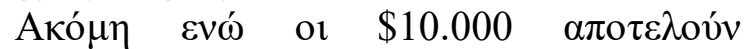

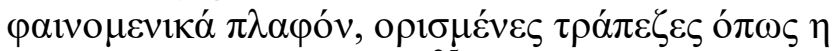

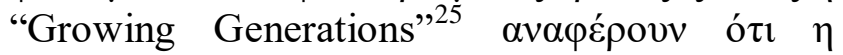

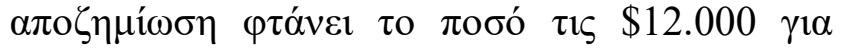

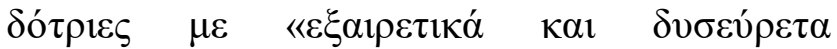

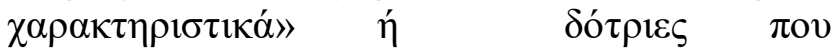
$\varepsilon \pi \alpha v \alpha \lambda \alpha \mu \beta \alpha ́ v o v v \tau \eta v \delta 1 \alpha \delta 1 \kappa \alpha \sigma i ́ \alpha$.

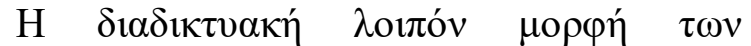

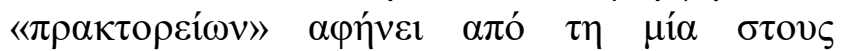

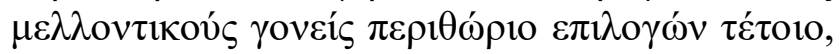

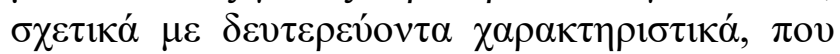

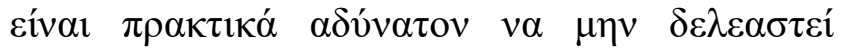

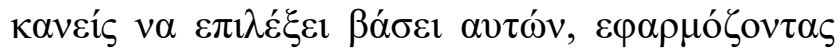

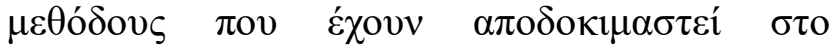

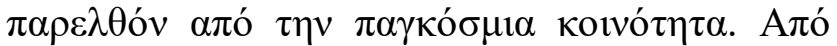

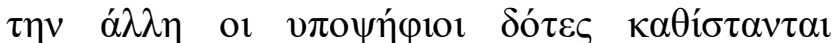

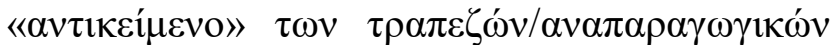

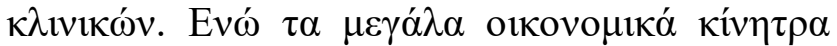

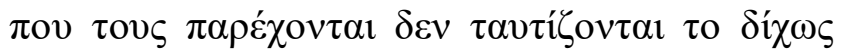

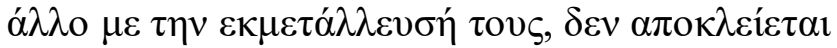

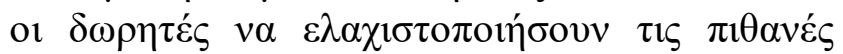

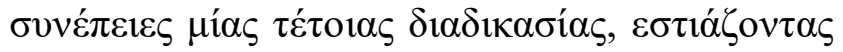

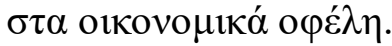

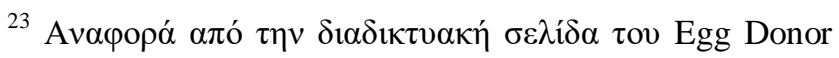
Center tov Dallas to 2008, $\beta \lambda$. http://www.eggdonorcenter.com/

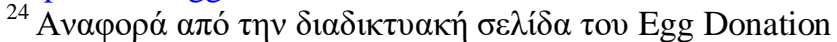
Inc. $\tau$ o 2008, $\beta \lambda$. http://www.eggdonor.com/

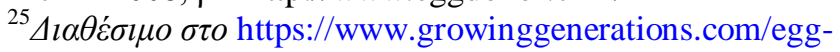
donor-program/egg-donors/egg-donor-pay/1
}

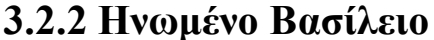

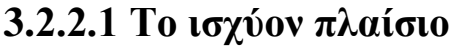

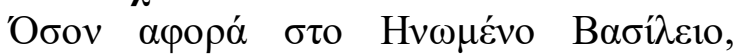

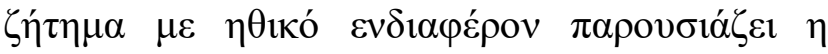
$\pi \mathrm{\circ} \lambda \iota \tau 1 \kappa \eta$ $\tau \eta \varsigma$ Human Fertilisation and Embryology Authority ( $\varepsilon \varphi \varepsilon \xi \eta \dot{n} \varsigma$ HFEA),

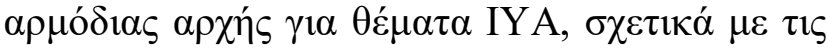

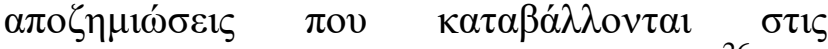
$\lambda \varepsilon \gamma o ́ \mu \varepsilon v \varepsilon \varsigma$ "egg-donors" $\kappa \alpha l$ "egg-sharers". ${ }^{26}$

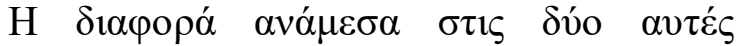

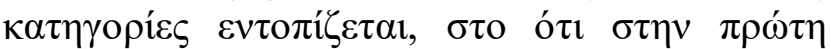

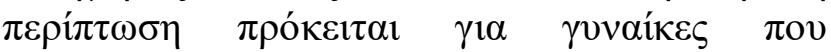

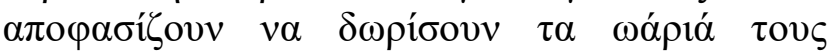

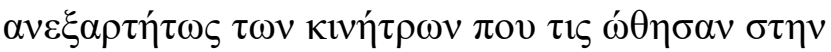

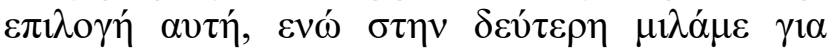

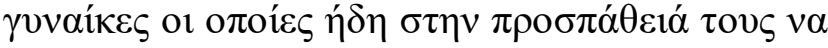

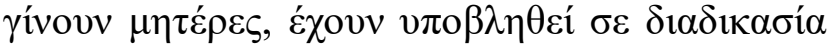

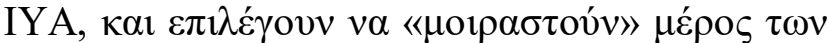

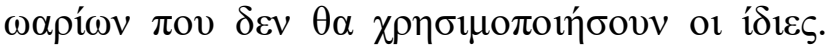

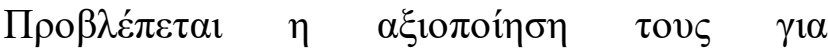

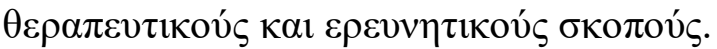

$\Gamma 1 \alpha \quad \kappa \alpha ́ \theta \varepsilon \quad \pi \varepsilon \rho i ́ \pi \tau \omega \sigma \eta \quad \pi \rho \circ \beta \lambda \varepsilon ́ \pi \varepsilon \tau \alpha 1$

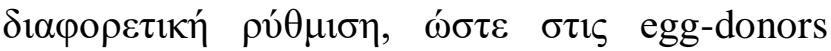

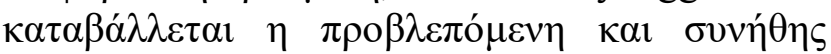
$\alpha \pi \circ \zeta \eta \mu i ́ \omega \sigma \eta$, evó or egg-sharers $\lambda \alpha \mu \beta \alpha ́ v o v v$ $\pi \alpha \rho \circ \chi \varepsilon \dot{\varepsilon} \varsigma \sigma \varepsilon \varepsilon \dot{\delta} \delta \circ \varsigma$.

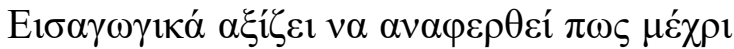

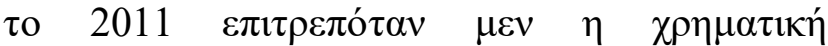

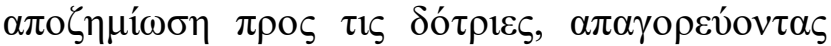

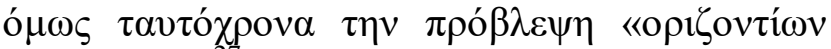

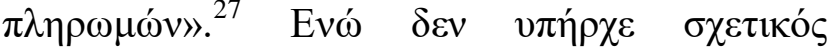

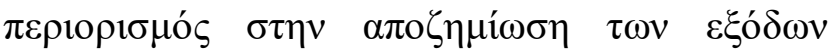

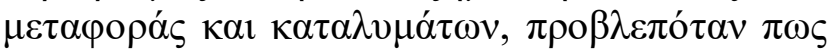

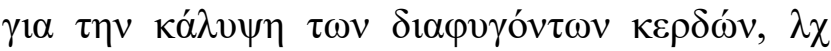

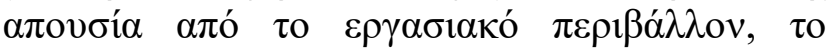

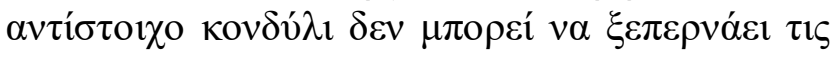

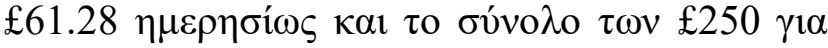

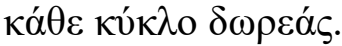

\footnotetext{
${ }^{26}$ Wilkinson S, Is the HFEA's Policy on Compensating Egg Donors and Egg Sharers Defensible? Med Law Review, 2013,21:173-212.

${ }^{27}$ HFEA, Code of Practice (8th edn October 2011 version) s.13.2.
} 


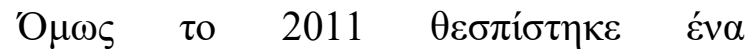

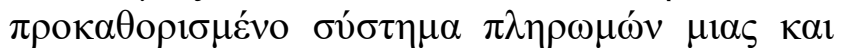

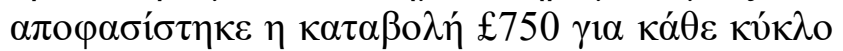

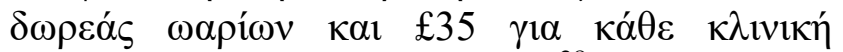

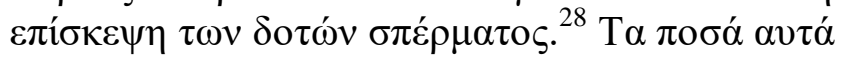

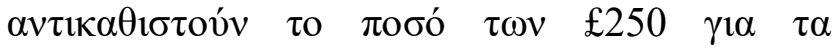

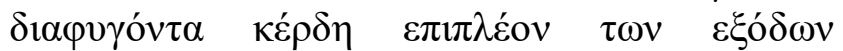

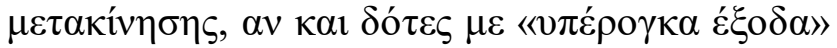

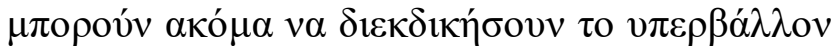

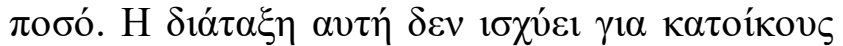

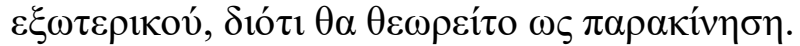

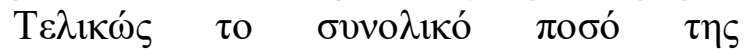

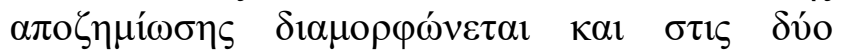

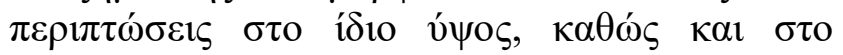

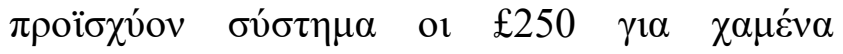

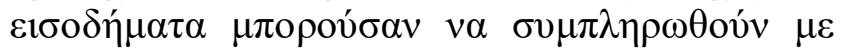

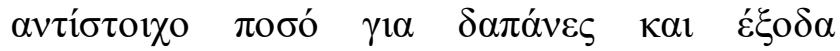

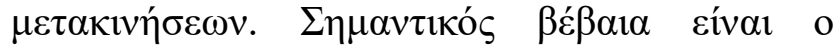

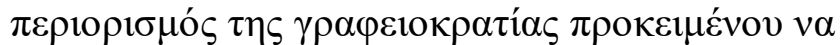

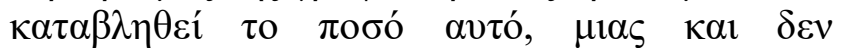

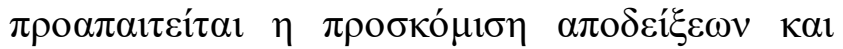

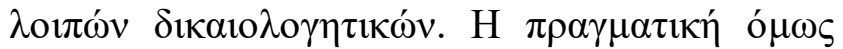

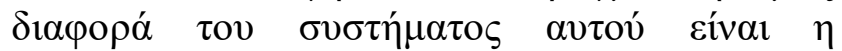

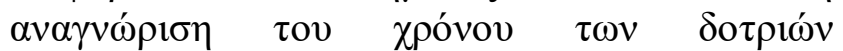

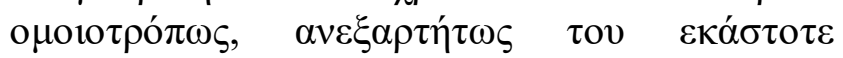

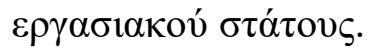

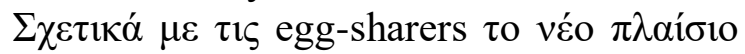

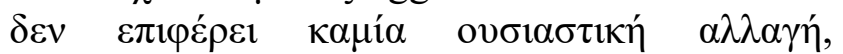

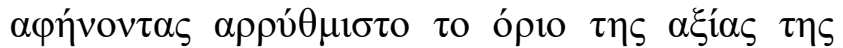

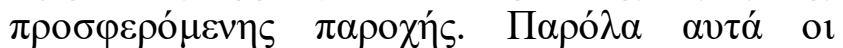

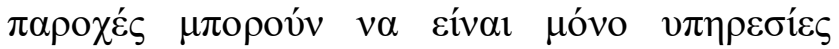

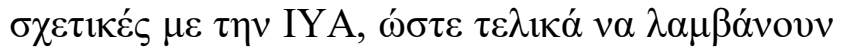
$\mu \varepsilon \gamma \alpha \dot{\alpha} \lambda \eta \quad \dot{\varepsilon} \kappa \pi \tau \omega \sigma \eta \quad \sigma \tau \eta v \quad \eta \dot{\delta} \eta \quad \lambda \alpha \mu \beta \alpha ́ v o v \sigma \alpha$

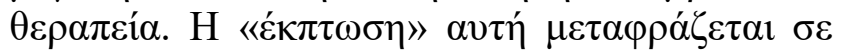

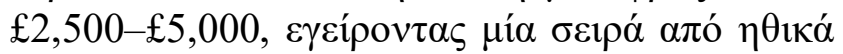

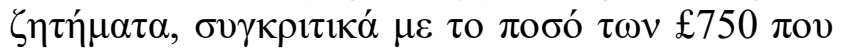
$\lambda \alpha \mu \beta a ́ v o v v$ ol egg-donors.

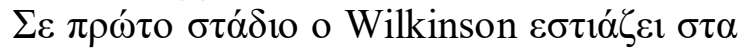

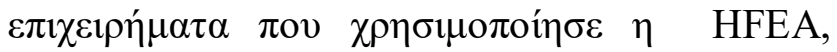

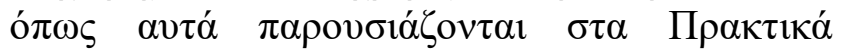

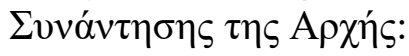

${ }^{28}$ HFEA, Authority Meeting Papers, Paper 612, 19 October 2011, 4.4.3.

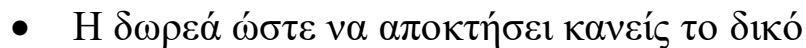

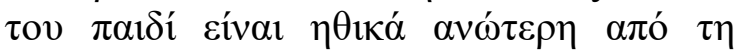

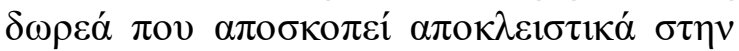

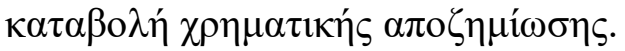

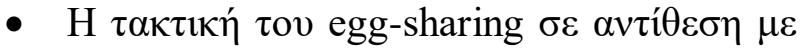

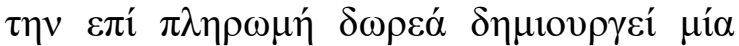
$\alpha i ́ \sigma \theta \eta \sigma \eta \alpha \lambda \lambda \eta \lambda \varepsilon \gamma \gamma$ vós.

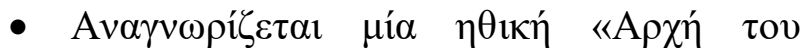

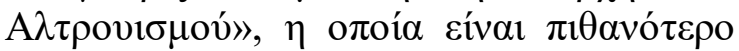

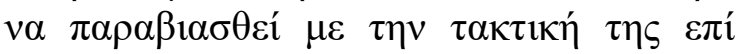

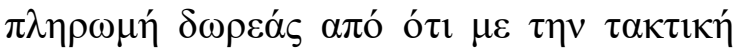

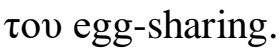

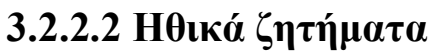

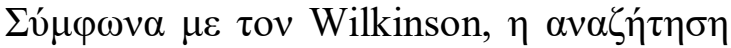

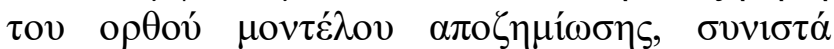

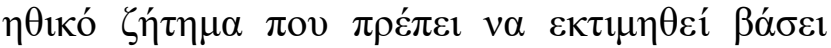

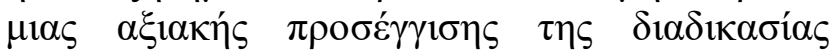

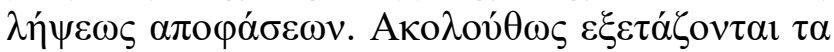
$\kappa \alpha ́ \tau \omega \theta \imath \varepsilon \pi \imath \chi \varepsilon \imath \rho \eta \dot{\mu} \mu \tau \alpha$ :

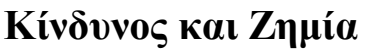

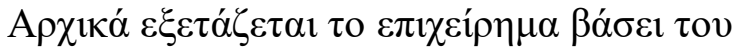

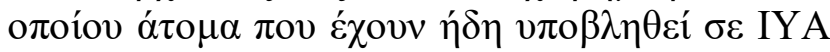

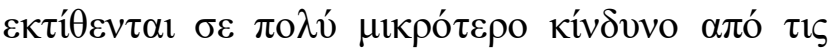

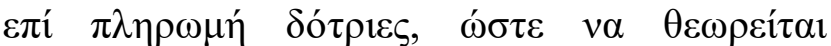

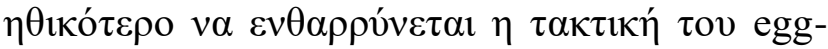

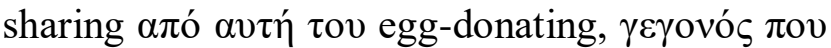

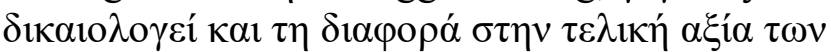

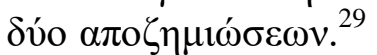

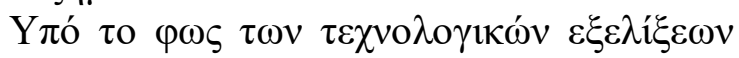

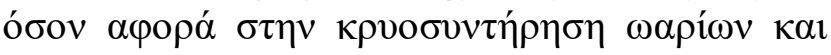

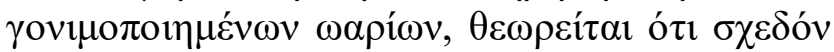

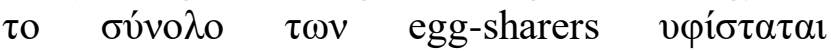

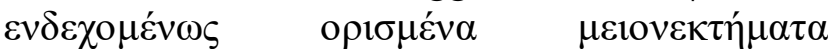

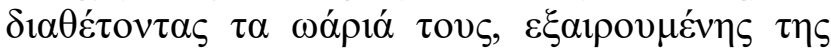
$\pi \varepsilon \rho i ́ \pi \tau \omega \sigma \eta \varsigma$ ó $\pi 0 v$ o $\varepsilon \pi \imath \theta v \mu \eta \tau o ́ s \quad \alpha \rho \imath \theta \mu o ́ \varsigma$

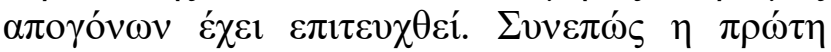
$\varepsilon \dot{v} \sigma \tau \alpha \sigma \eta \quad \sigma \tau o \quad \pi \rho 0 \alpha v \alpha \varphi \varepsilon \rho \theta \varepsilon \dot{\varepsilon} v \quad \varepsilon \pi \imath \varepsilon \varepsilon i ́ p \eta \mu \alpha$

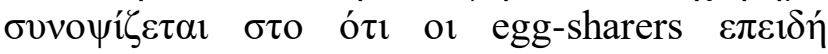

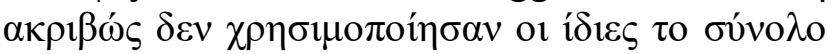

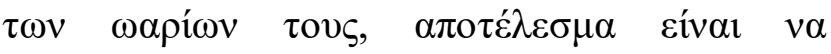

29 HFEA, Authority Meeting Papers, Paper 612, 19 October 2011, 2.3.3. 


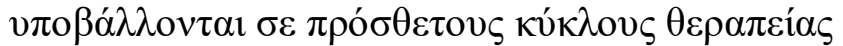

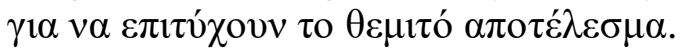

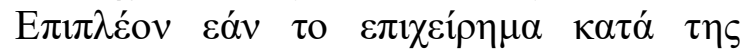

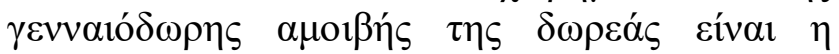

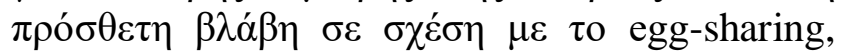

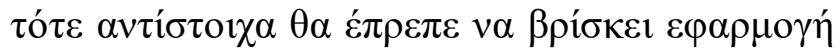

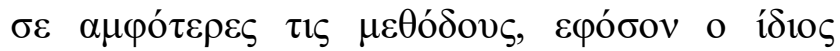

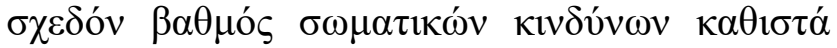

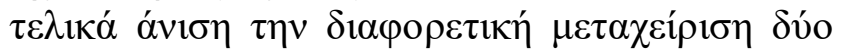

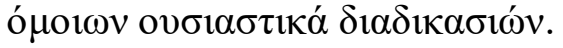

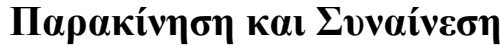

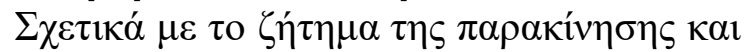

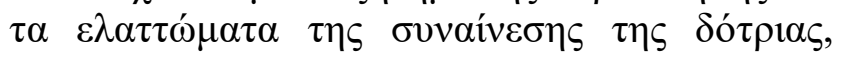

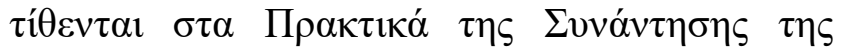

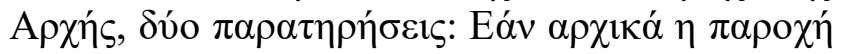

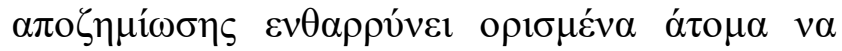

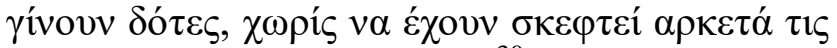

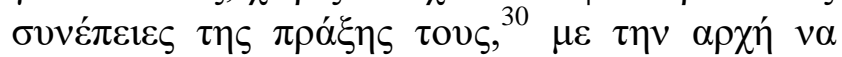

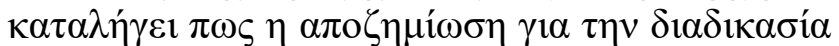

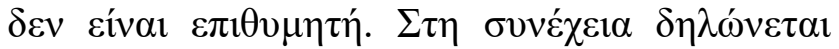

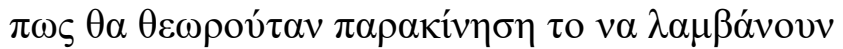

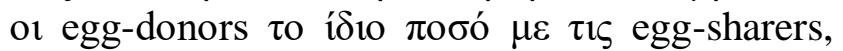
$\delta \eta \lambda \alpha \delta \eta ் ~ £ 2500-£ 5000$.

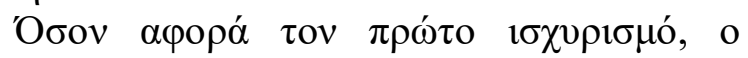

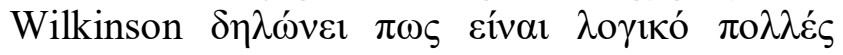

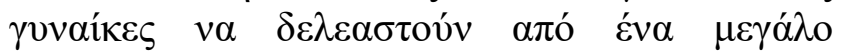

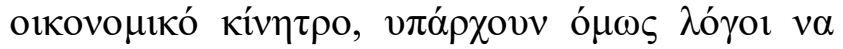

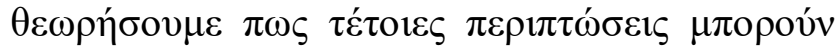

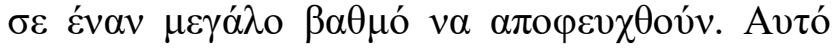

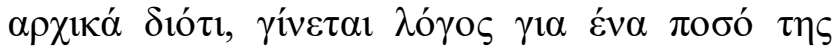

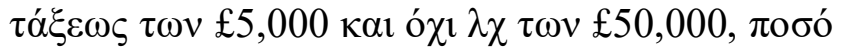

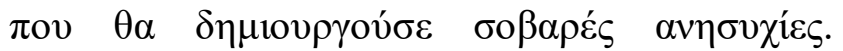

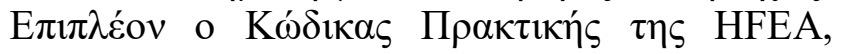

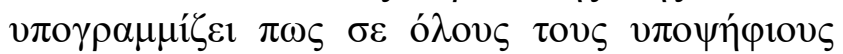

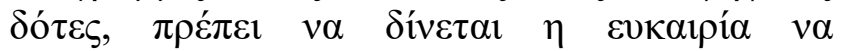

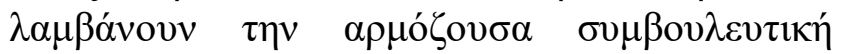

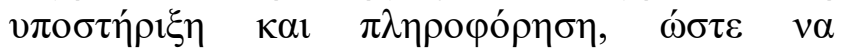

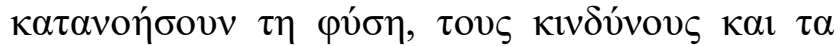

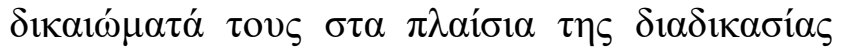

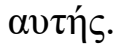

${ }^{30}$ HFEA, Authority Meeting Papers, Paper 612, 19 October 2011, 5.1.6.

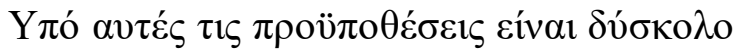

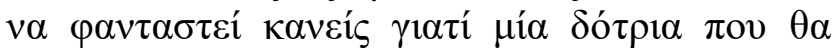

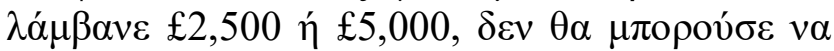

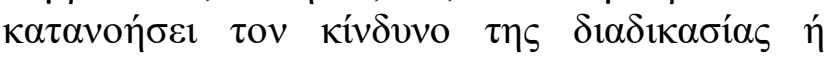

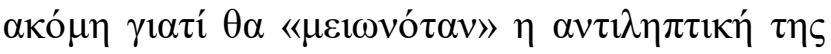

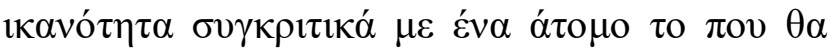

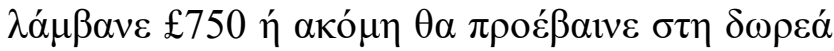
$\varepsilon v \tau \varepsilon \lambda \omega ́ \varsigma \alpha \lambda \tau \rho o v i \sigma \tau \imath \kappa \alpha ́$.

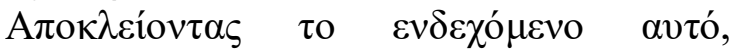

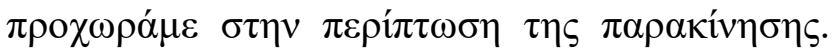

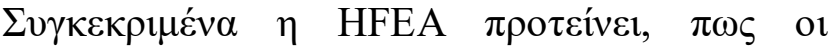

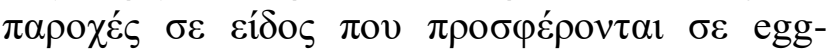

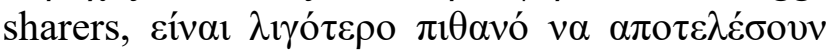

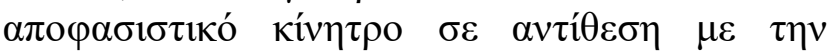

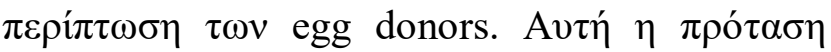

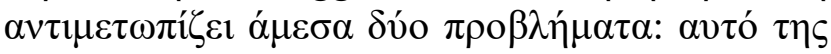

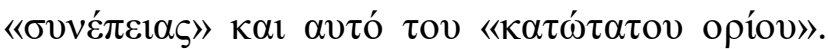

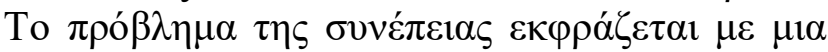

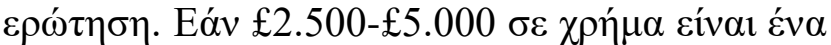

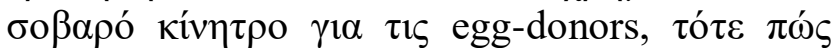

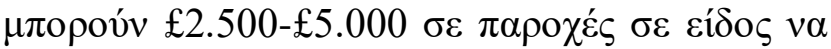

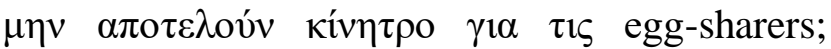

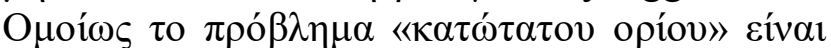

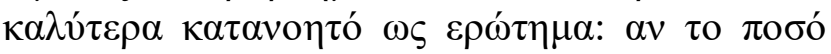

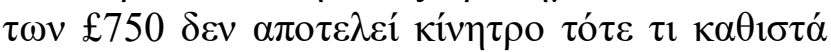

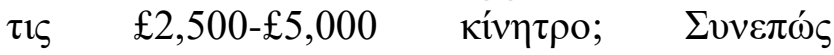

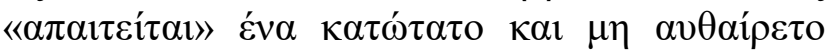

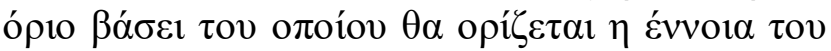

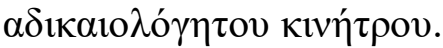

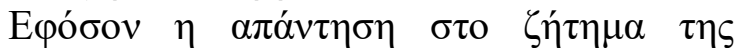

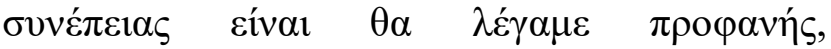

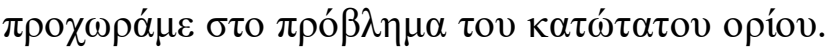

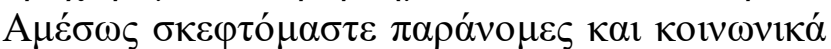

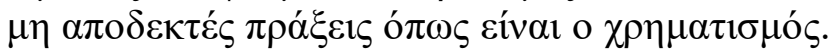

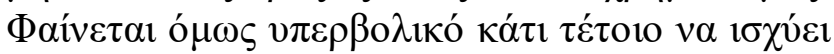

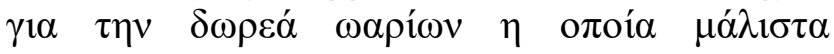

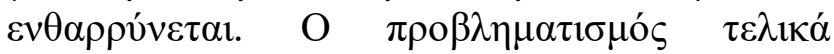

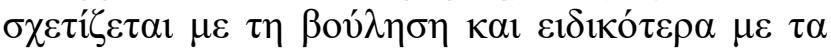

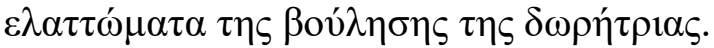

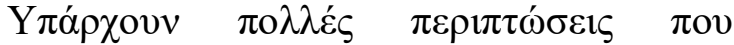

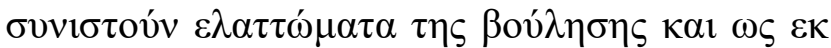

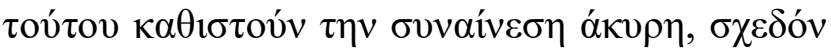

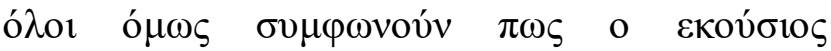

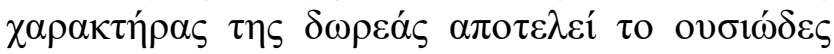




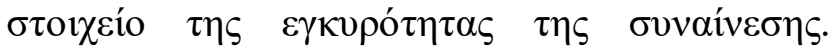

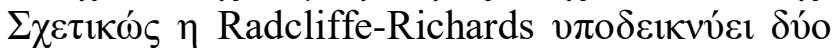

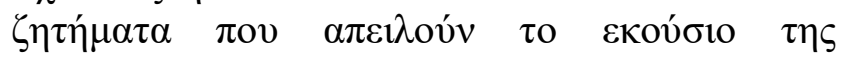

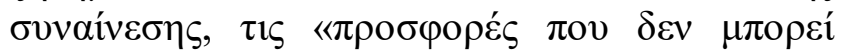

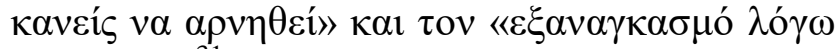

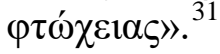

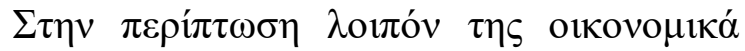

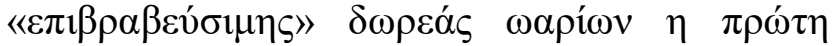

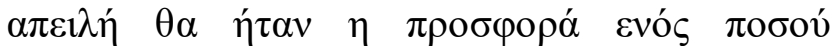

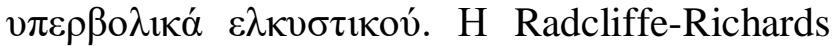

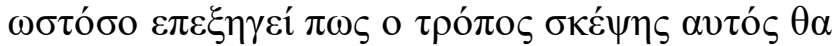

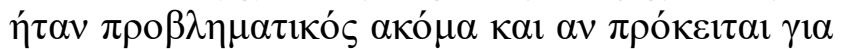

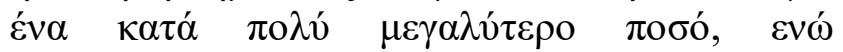

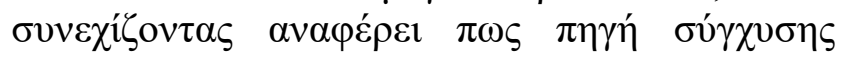

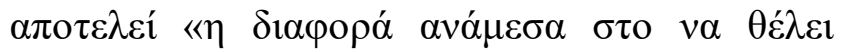

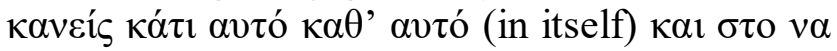

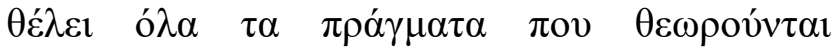

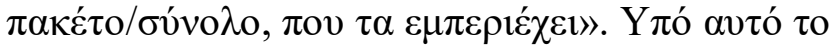

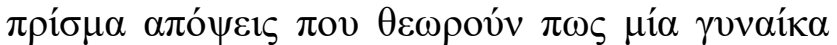

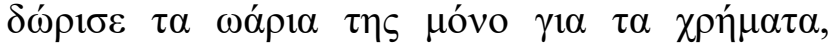

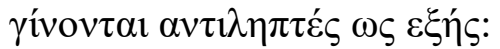

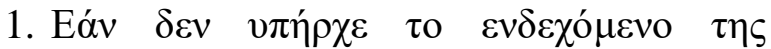

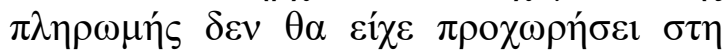
$\delta \omega \rho \varepsilon \alpha ́$.

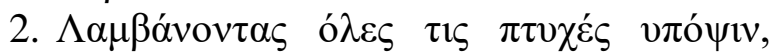

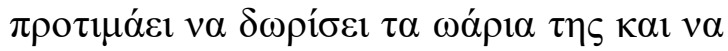

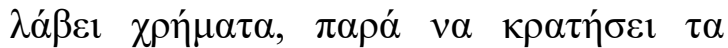

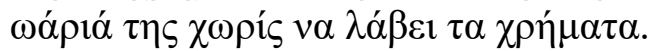

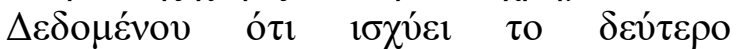

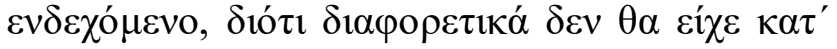

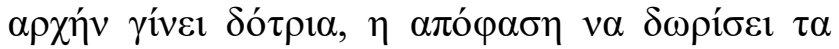

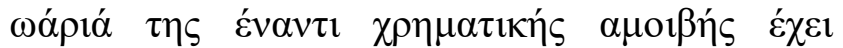

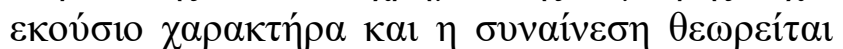

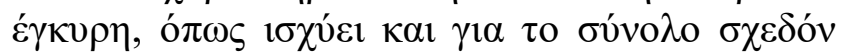
$\tau \omega v \alpha \pi \circ \varphi \alpha ́ \sigma \varepsilon \omega v \mu \alpha \varsigma$.

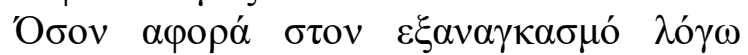

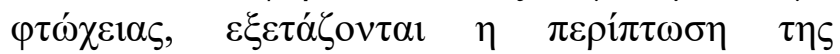

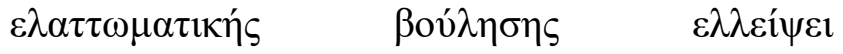

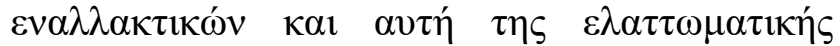

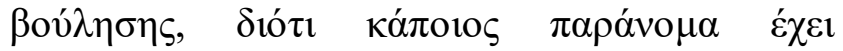

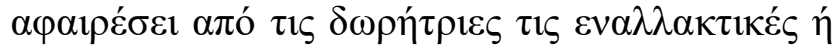

\footnotetext{
${ }^{31}$ Radcliffe Richards J. Consent with Inducements: the Case of Body Parts and Services, The Ethics of Consent: Theory and Practice, OUP,2006,281-291.
}

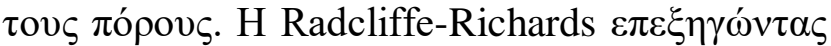

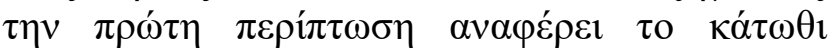
$\pi \alpha \rho \alpha ́ \delta \varepsilon \imath \gamma \mu \alpha: \quad « \Sigma \tau \eta \nu \quad \pi \varepsilon \rho i ́ \pi \tau \omega \sigma \eta \quad \varepsilon v o ́ \varsigma$

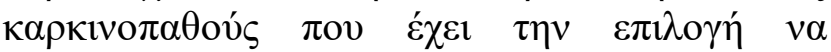

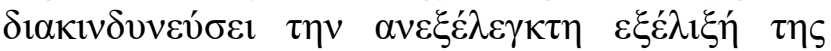

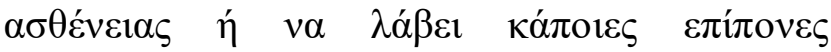

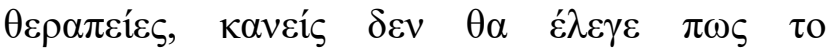

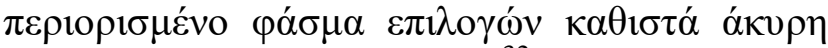

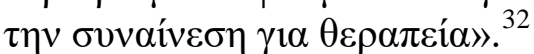

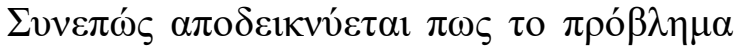

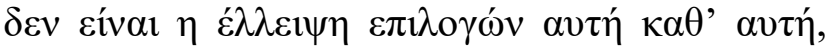

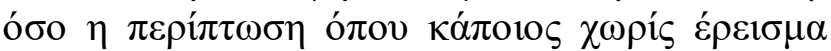

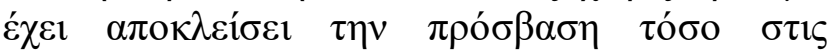

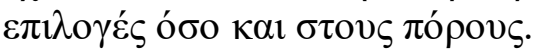

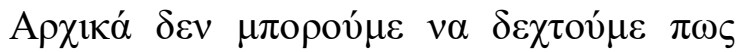

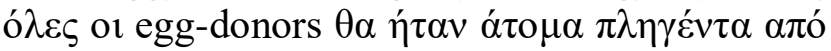

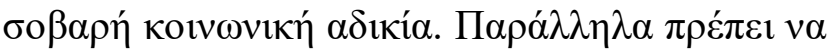

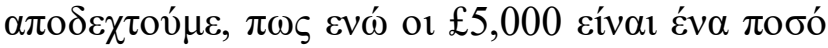

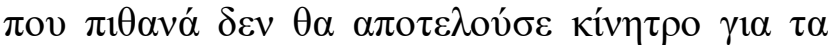

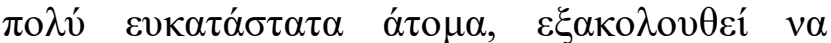

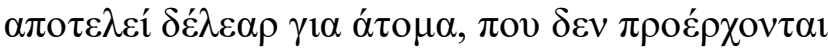

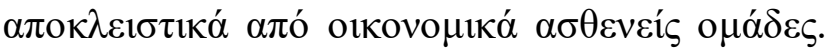

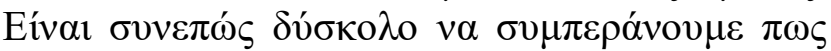

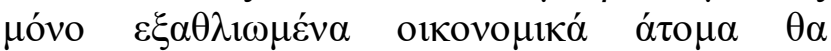

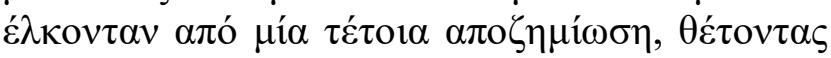

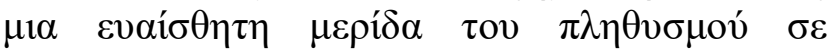

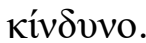

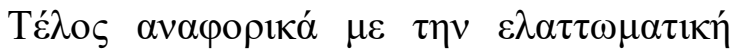

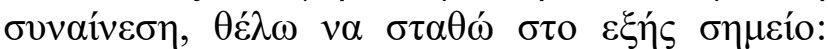

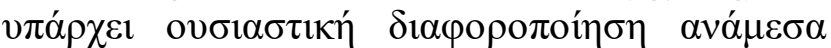

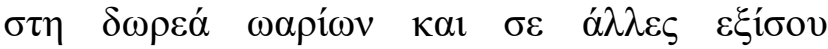

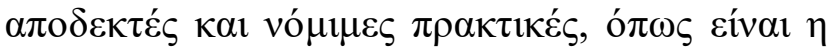

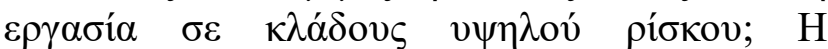

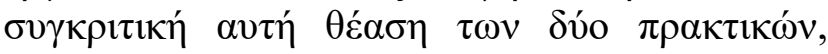

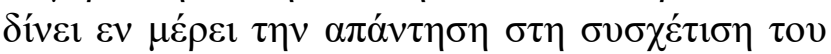

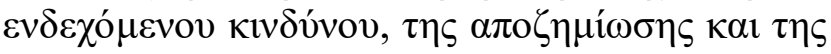

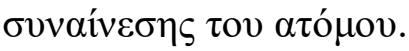

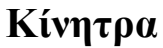

$\mathrm{H} \delta 1 \alpha \beta$ ov́$\lambda \varepsilon v \sigma \eta \quad \tau \eta \varsigma$ HFEA,

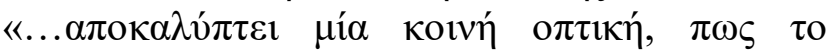

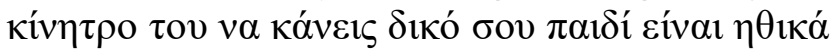

\footnotetext{
${ }^{32}$ Ibidem
} 


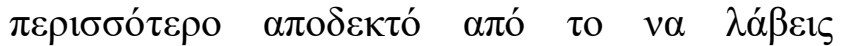
$\chi \rho \eta \dot{\mu} \mu \tau \alpha \ldots »$.

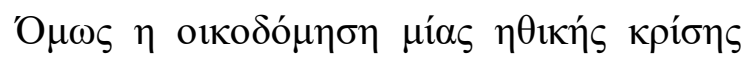

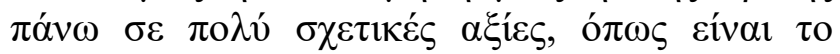

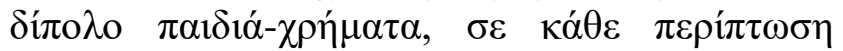

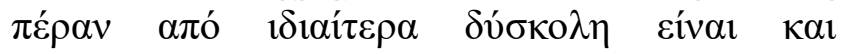

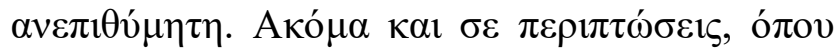
$\sigma \varepsilon \quad \pi \alpha \gamma \kappa o ́ \sigma \mu 10 \quad \varepsilon \pi i ́ \pi \varepsilon \delta$ o

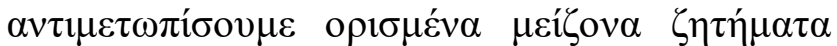

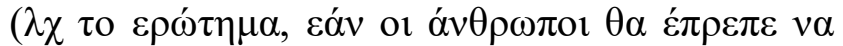

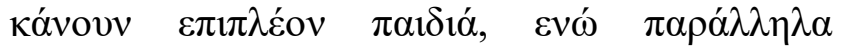

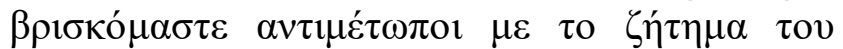

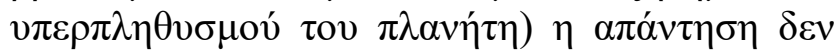

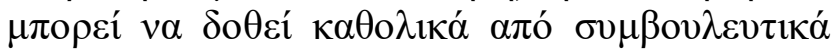
$\sigma \omega ́ \mu \alpha \tau \alpha$ ó $\pi \omega \varsigma \eta$ HFEA $\kappa \alpha \theta \imath \tau \tau \omega ́ v \tau \alpha \varsigma \alpha \pi \alpha \rho \alpha ́ \delta \varepsilon \kappa \tau \eta$

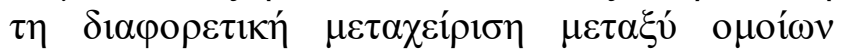
o $\mu \alpha ́ \delta \omega v$.

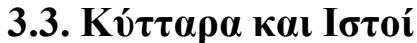

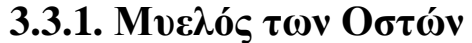

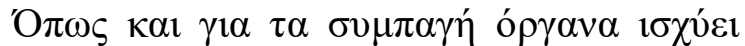

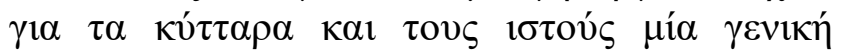

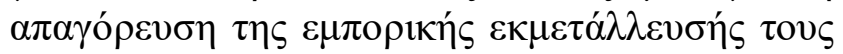
( $\beta \lambda$ Oviedo Convention, Human Tissue Act,

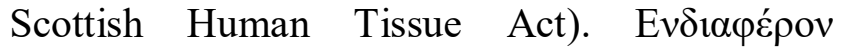

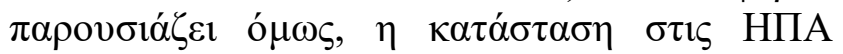

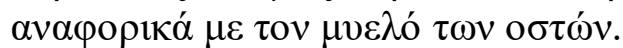

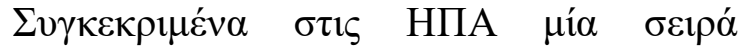

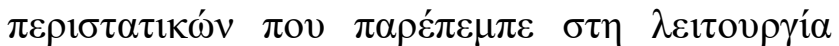

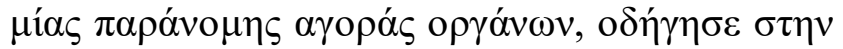
$\theta \dot{\varepsilon} \sigma \pi \imath \sigma \eta \tau \eta \varsigma$ National Organ Transplantation Act

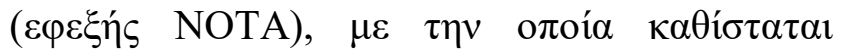
$\pi \alpha \rho \alpha ́ v o \mu \eta \quad \eta \quad \varepsilon \mu \pi$ о

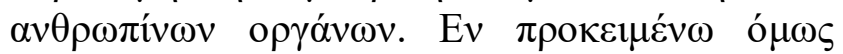

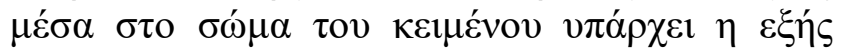

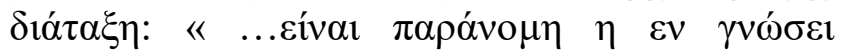

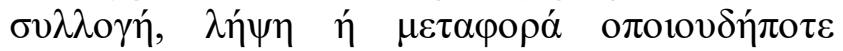

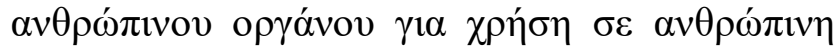

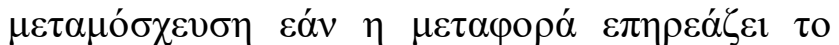

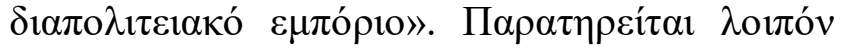

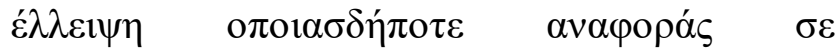

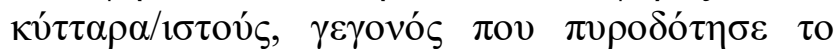

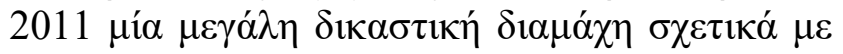

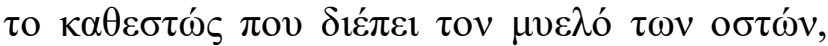

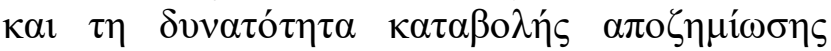

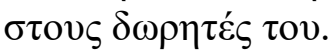

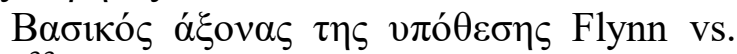

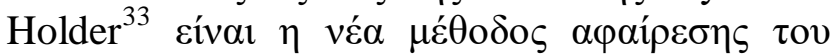

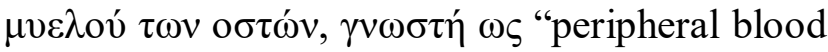

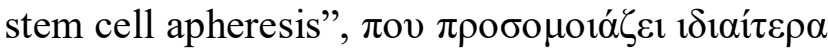

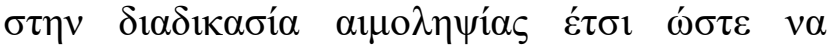

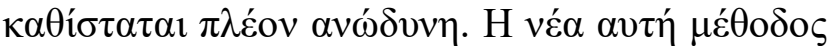

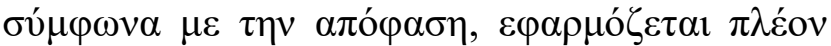

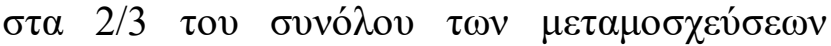

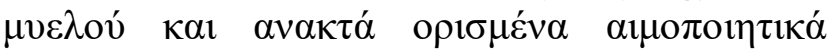

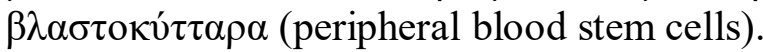

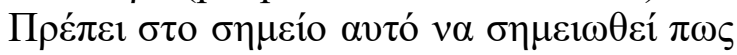
$\eta \varepsilon \pi ı \tau v \chi \eta \mu \varepsilon ́ v \eta \mu \varepsilon \tau \alpha \mu o ́ \sigma \chi \varepsilon v \sigma \eta \mu v \varepsilon \lambda o v ́, \varepsilon \xi \alpha \rho \tau \alpha ́ \tau \alpha 1$

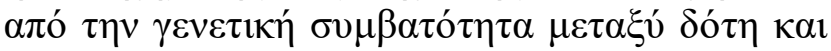

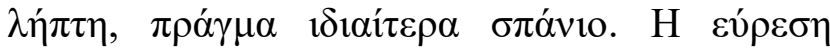

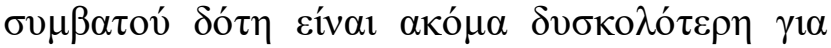

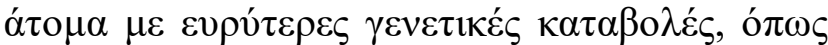

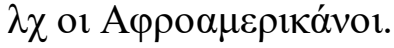

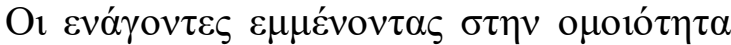

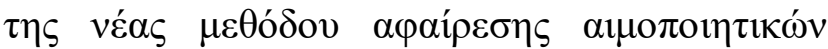
$\beta \lambda \alpha \sigma \tau о \kappa v \tau \tau \alpha ́ \rho \omega v \quad \mu \varepsilon \quad \tau \eta v \quad \alpha \mu о \lambda \eta \psi i ́ \alpha$,

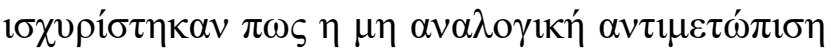

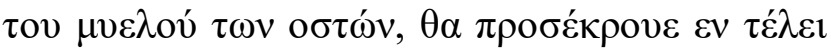

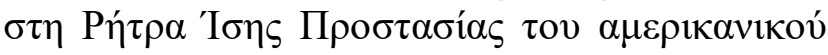

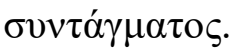

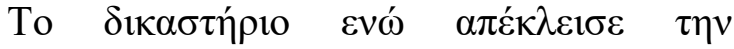

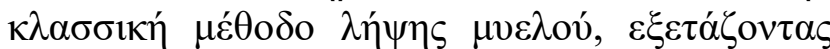

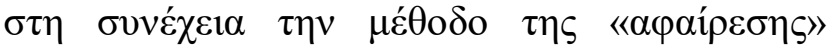

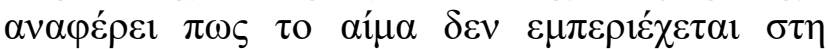

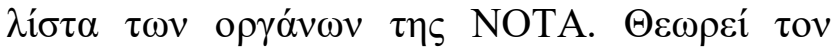

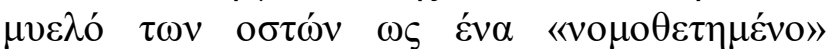

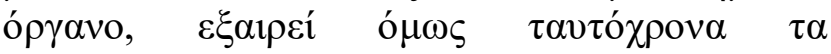

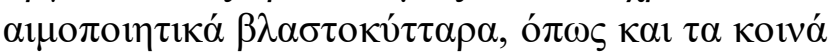

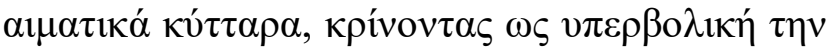

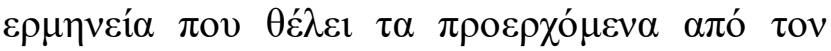

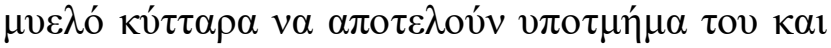

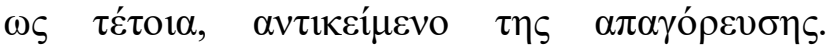

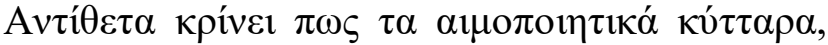

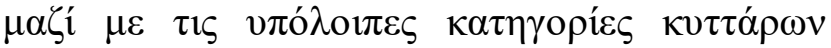

\footnotetext{
${ }^{33} \Delta \iota \alpha \theta \dot{\varepsilon} \sigma \iota \mu о \sigma \tau о$

http://cdn.ca9.uscourts.gov/datastore/opinions/2011/12/01/ 10-55643.pdf
} 


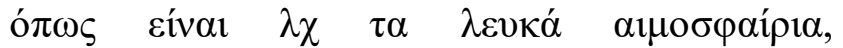

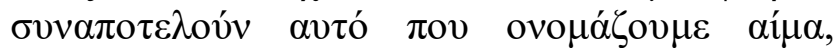

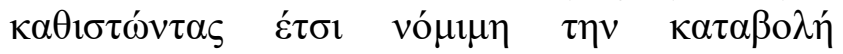
$\alpha \pi \circ \zeta \eta \mu \omega ́ \sigma \varepsilon \omega \varsigma$.

\section{4. $\Sigma v \mu \pi \varepsilon \rho \alpha ́ \sigma \mu \alpha \tau \alpha$}

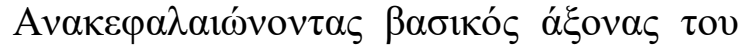

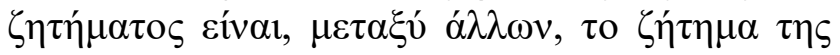

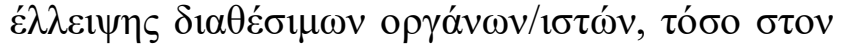

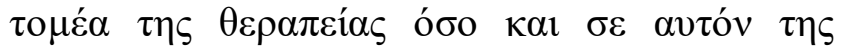

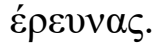

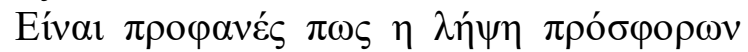

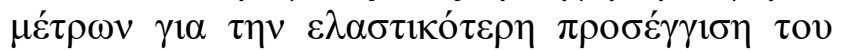

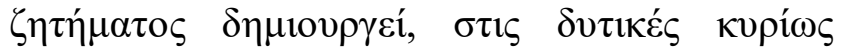

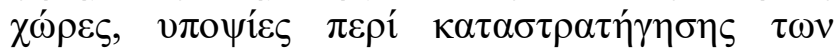

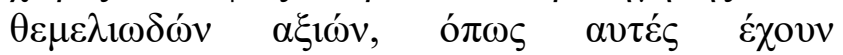

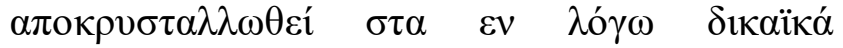

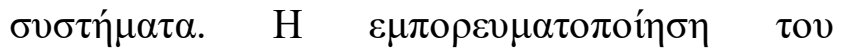

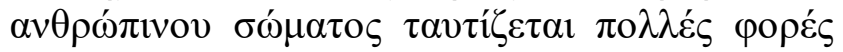

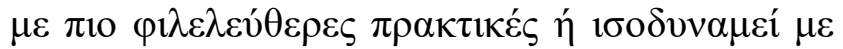

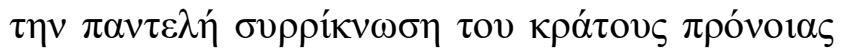

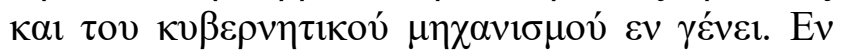

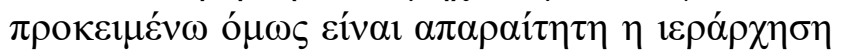
$\tau \omega v \quad \pi \rho \circ \beta \lambda \eta \mu \alpha ́ \tau \omega v, \quad \pi 0 v \quad \kappa \alpha \lambda \varepsilon i ́ \tau \alpha l \quad v \alpha$

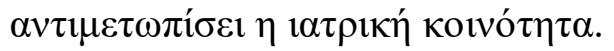

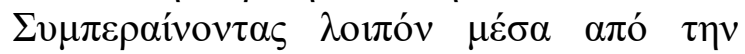

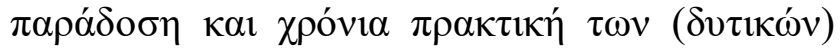

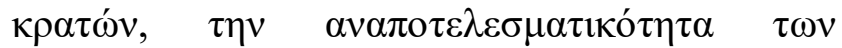

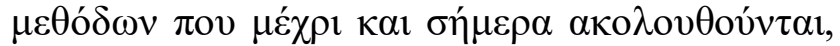

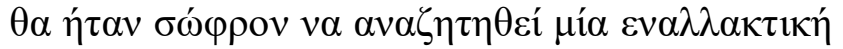

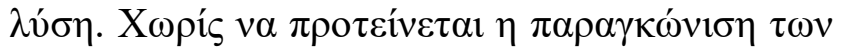

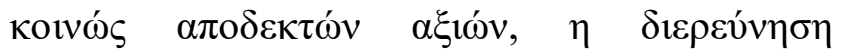

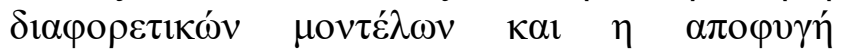

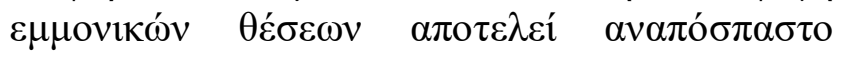

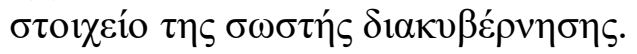

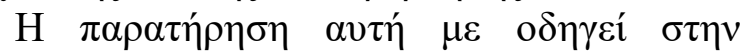

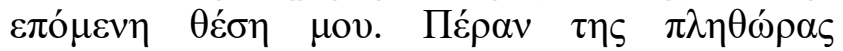

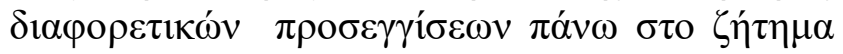

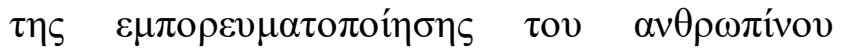

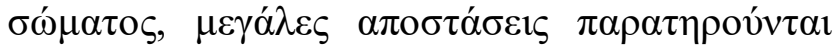

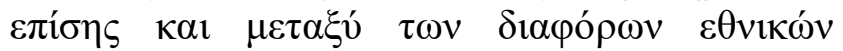

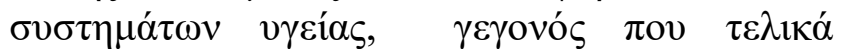

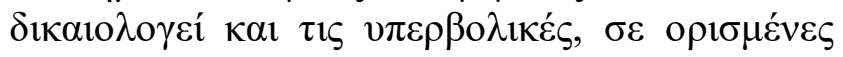

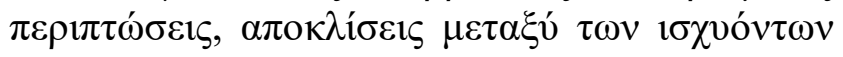

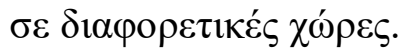

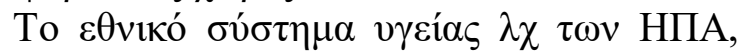

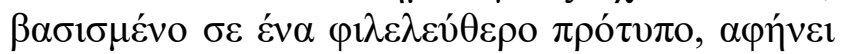

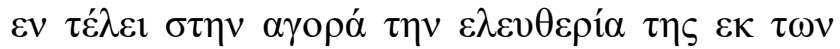

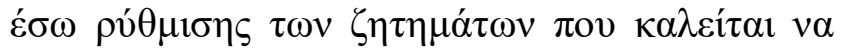

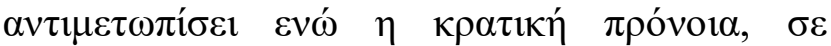

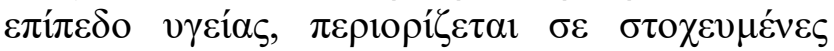
$\delta \rho \alpha ́ \sigma \varepsilon ı \varsigma$ ( $\beta \lambda$ Medicaid, Medicare).

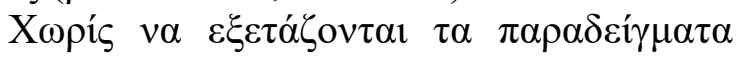

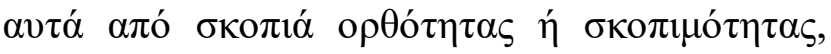

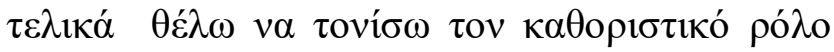

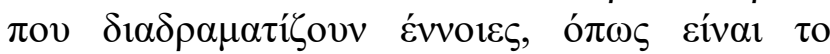

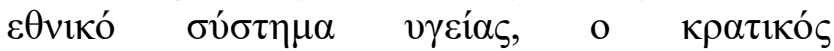

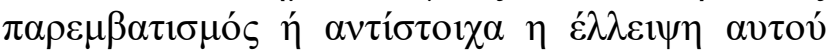

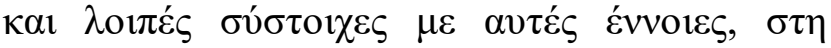

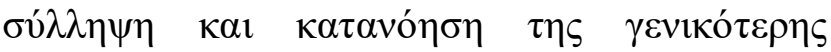

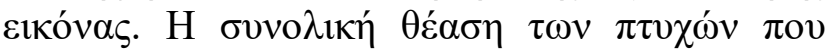

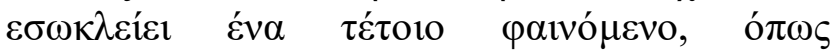

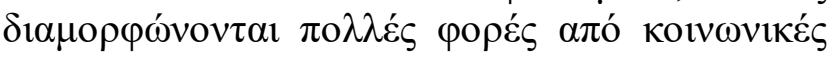

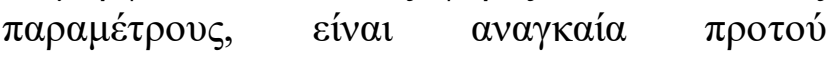

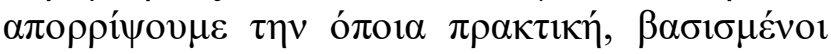

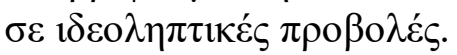

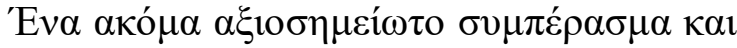

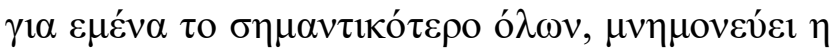

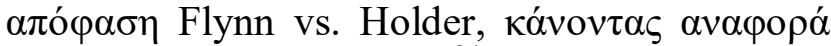

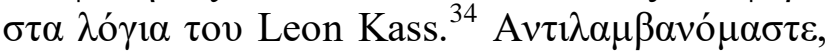

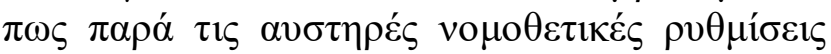

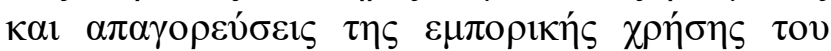

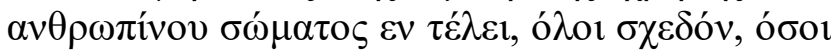

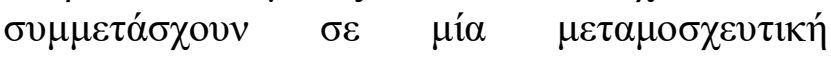

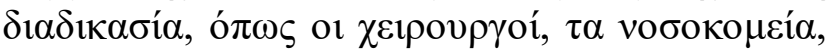

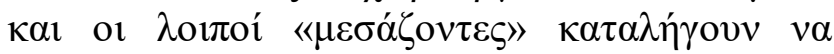

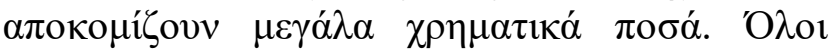

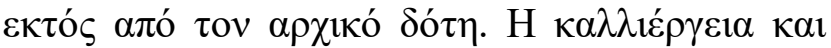

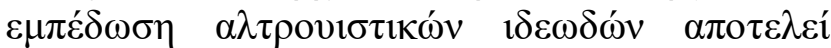

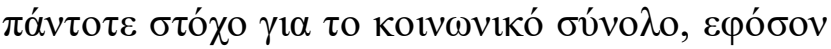

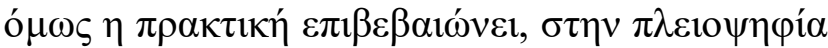

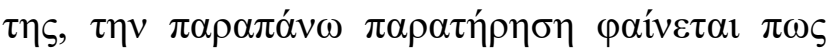

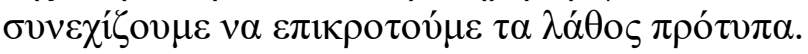

${ }^{34}$ Kass L, Life. Liberty and the Defense of Dignity: The Challenge for Bioethics, Encounter Books, 2002. 


\section{BIOHOIKA}

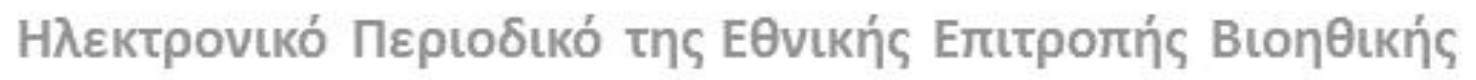

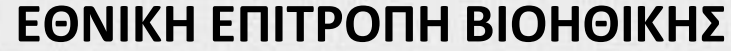

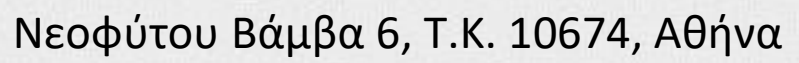

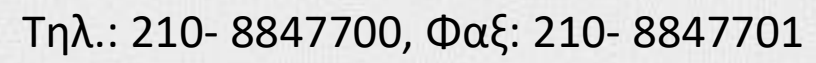

E-mail: secretariat@bioethics.gr

url: www.bioethics.gr

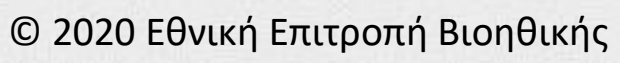

ISSN: $2653-8660$ 